\title{
Nanoscale structural organization and stoichiometry of the budding yeast kinetochore
}

\author{
Konstanty Cieslinski ${ }^{1,2}$, Yu-Le $\mathrm{Wu}^{1,3}$, Lisa Nechyporenko ${ }^{1,4}$, Sarah Janice Hörner ${ }^{1,5}$, Duccio Conti ${ }^{6}$,
} Jonas Ries ${ }^{1, *}$

1. European Molecular Biology Laboratory, Cell Biology and Biophysics Unit, Heidelberg, Germany

2. DKFZ, Translational Radiation Oncology Unit, Heidelberg, Germany

3. Collaboration for joint PhD degree between EMBL and Heidelberg University, Faculty of Biosciences, Heidelberg, Germany

4. Institute of Pharmacy and Molecular Biotechnology, Heidelberg University, Heidelberg, Germany

5. Institute of Molecular and Cell Biology, Mannheim University of Applied Sciences and Interdisciplinary Center for Neuroscience, Heidelberg University, Heidelberg, Germany

6. Department of Mechanistic Cell Biology, Max Planck Institute of Molecular Physiology, Dortmund, Germany

* Correspondence: jonas.ries@embl.de

\begin{abstract}
Proper chromosome segregation is crucial for cell division. In eukaryotes, this is achieved by the kinetochore, an evolutionarily conserved multi-protein complex that physically links the DNA to spindle microtubules, and takes an active role in monitoring and correcting erroneous spindlechromosome attachments. Our mechanistic understanding of these functions, and how they ensure an error-free outcome of mitosis, is still limited, partly because we lack a comprehensive understanding of the kinetochore structure in the cell. In this study, we use single molecule localization microscopy to visualize individual kinetochore complexes in situ in budding yeast. For all major kinetochore proteins, we measured abundance and position within the metaphase kinetochore. Based on this comprehensive dataset, we propose a quantitative model of the budding yeast kinetochore. While confirming many aspects of previous reports based on bulk imaging of kinetochores, our results present a somewhat different but unifying model of the inner kinetochore. We find that the centromere-specialized histone Cse4 is present in more than two copies per kinetochore along with its binding partner Mif2.
\end{abstract}

\section{Introduction}

Cell division is a process of paramount importance for organismal life, ultimately ensuring the faithful propagation of the genome in space and time. Erroneous chromosome segregation can lead to aneuploidy, where daughter cells receive an aberrant karyotype which, in turn, can result in dramatic developmental defects or cell death (Santaguida and Amon, 2015). A macromolecular complex called kinetochore assembles at the centromere of each sister chromatid to generate robust connections between chromosomes and spindle microtubules (reviewed in (Musacchio and Desai, 2017)). The general architecture of the kinetochore is conserved in all eukaryotes (Drinnenberg et al., 2016; Hooff et al., 2017). A simple model to study its properties is Saccharomyces cerevisiae, where the kinetochore assembles from one nucleosome and is attached to one microtubule (Winey et al., 1995). Conversely, multiple copies of units analogous to the budding yeast kinetochore bind to many 
microtubules in other fungi and multicellular organisms (Musacchio and Desai, 2017; Zinkowski et al., 1991). The kinetochore takes part in several processes during mitosis including maintaining proper chromosome attachment to the spindle, translating the pushing-pulling forces into movement and controlling the mitotic progression trough the spindle assembly checkpoint (Aravamudhan et al., 2015; Asbury, 2017; Joglekar et al., 2010). These functions are strongly dependent on the kinetochore's structure and its potential remodeling over the cell cycle (Conti et al., 2017; Dhatchinamoorthy et al., 2017; Joglekar et al., 2009).

Early electron microscopy studies defined three electron-dense regions in the kinetochore - the inner kinetochore, the outer kinetochore, and the fibrous corona (Rieder, 1982). In S. cerevisiae, where the corona is absent, the inner kinetochore includes the centromeric nucleosome containing an $\mathrm{H} 3$ variant called Cse4, the CBF3 subcomplex (Cep3, Ndc10, Ctf13 and Skp1), the Mif2 and Cnn1 module (Cnn1, Ctf3, Wip1, Mcm16/22, Mhf1/2), Nkp1/2, the COMA subcomplex (Ctf19, Okp1, Mcm21, Ame1), and Chl4/Iml3. The outer kinetochore consists of the microtubule-interacting unit built by Spc105, the MIND subcomplex (Mtw1, Dsn1, Nnf1, Ns11), the NDC80 subcomplex (Ndc80, Spc24, Spc25, Nuf2) and the DAM1 ring (Musacchio and Desai, 2017) (Figure 1A). Human counterparts are shown in Figure $1 \mathrm{~A}$ in the upper right corner of each protein.

Despite advances in the last decades in understanding kinetochore composition, a complete picture of its organization in cells is still unclear. A significant portion of structured components of both human and budding yeast kinetochores have been already crystallized (for an overview see Dimitrova et al., 2016; Hinshaw and Harrison, 2019; Jenni and Harrison, 2018; Musacchio and Desai, 2017; Yan et al., 2019). Most of the structural information regarding the full yeast kinetochore comes from electron microscopy (EM) and fluorescence microscopy studies. EM studies revealed the overall shape of the budding yeast kinetochore (Gonen et al., 2012; McIntosh et al., 2013), but could not assign most proteins in the electron density maps (Hinshaw and Harrison, 2019, p. 2; Yan et al., 2019). On the other hand, conventional fluorescence microscopy has provided information about the position of several kinetochore components along the spindle axis (Aravamudhan et al., 2014; Haase et al., 2013; Joglekar et al., 2009). However, this approach can only reveal the structural average of all kinetochores, because individual complexes are smaller than the resolution limit of conventional light microscopy (approximately $250 \mathrm{~nm}$ (Abbe, 1873)) and clustered. As a result, in budding yeast all 16 kinetochores are observed as one (during interphase) or two fluorescent spots (mitosis) (Joglekar et al., 2006), and fine structural details of individual kinetochores cannot be observed. Thus, a comprehensive understanding of the structure of the kinetochore is still missing.

In the budding yeast kinetochore, built on a short centromere (approximately $125 \mathrm{bps}$ ) (Clarke and Carbon, 1980), microtubules are captured by multiple copies of the NDC80 and DAM1 complexes. Precisely how many complexes are present, however, remains controversial, with estimates ranging significantly. To examine this question, previous studies used fluorescence microscopy to quantify the absolute copy numbers of the major kinetochore components. In this approach the protein of interest was tagged with a suitable fluorescent protein. The relative brightness of the observation was then compared to a reference protein tagged with the same fluorophore (Joglekar et al., 2006, 2008; Lawrimore et al., 2011; Dhatchinamoorthy et al., 2017). These studies generally agreed that the outer kinetochore proteins are the most abundant and the inner kinetochore proteins the least abundant. Ndc80 has been shown to be present in 6 to 19 copies per kinetochore. Smaller or equal amounts were found for the MIND complex (4 to 7 copies) and Spc105 (4 to 5 copies). The COMA complex was shown to be present in 2 to 5 copies. Within the inner kinetochore, Cep3 was found to have 2 to 3.4 copies, Mif2 2 to 3.6 copies, and Cnn1 and Cse 42 to 6 copies (Shivaraju et al., 2012; Wisniewski 
et al., 2014). The differences among the results may arise from the choice of the counting reference, cell cycle stage, fluorescent protein, method and optical system used (Joglekar et al., 2008). Such large discrepancies prevent generating a detailed structural model. Open fundamental questions include: How do the Mif2 and Cnn1 assembly pathways quantitatively contribute to the copy number of NDC80? How many COMA complexes exist within the buddying yeast kinetochore?

Another extensively debated question in the field is the exact stoichiometry at centromeres of the histone protein Cse4. (Clarke and Carbon, 1980; Keith and Fitzgerald-Hayes, 2000; Ng and Carbon, 1987). To date, a series of alternative structures have been proposed to define the nature of the centromeric nucleosome. These hypotheses include hemisome (Bui et al., 2012; Dalal et al., 2007), hexameric (Mizuguchi et al., 2007) or octameric configurations (Camahort et al., 2009), where a single or two copies of Cse4 are present (Black and Cleveland, 2011). With regards to Cse4 copy number biochemical approaches have reported the presence of a single Cse4 nucleosome at centromeres (Furuyama and Biggins, 2007; Krassovsky et al., 2012). In contrast, in vivo studies showed a high variability of Cse4 copy number per kinetochore, ranging from 2 (Dhatchinamoorthy et al., 2017; Shivaraju et al., 2012; Wisniewski et al., 2014) up to 4 - 6 copies (Lawrimore et al., 2011). Interestingly, also the very first SMLM-based counting of Cnp1, the Cse4 homologue in fission yeast, reported 6 - 7 Cnp1 copies per spindle microtubule (Lando et al., 2012; Camahort et al., 2009). Therefore, the identity and copy number of the centromeric nucleosome is still an unanswered question in the centromere and kinetochore fields.

Super-resolution microscopy, and specifically Single-Molecule Localization Microscopy (SMLM) (Betzig et al., 2006; Hess et al., 2006; Rust et al., 2006), achieves nanometer resolution combined with molecular specificity, and has the potential to bridge this gap in our knowledge. It has been used to get structural insights into the organization of multi-protein complexes such as the nuclear pore complex (Szymborska et al., 2013), the endocytic machinery (Mund et al., 2018; Sochacki et al., 2017), centrioles (Sieben et al., 2018) or synaptic proteins (Dani et al., 2010). In this study, we use SMLM to determine the location of key proteins and their copy numbers with single kinetochore resolution in $S$. cerevisiae cells (Figure 1). From these data, we generated a comprehensive model of how the major components are positioned and what their stoichiometry is in the budding yeast metaphase kinetochore in situ.

\section{Results}

\section{Individual kinetochores can be observed with SMLM}

In order to determine whether SMLM can be used to visualize individual kinetochores, we imaged yeast cells in which Ndc80 was endogenously tagged with mMaple, and Spc42 (spindle pole body) with GFP (Figure 1B). When we imaged unsynchronized cells, we observed that in interphase cells all kinetochores are packed within a small cluster with a size below the resolution limit of standard microscopy, with the tendency to organize into a rosette-like configuration similar to what is observed in human cells in early prometaphase (Figure 1B) (Chaly and Brown, 1988; Jin et al., 2000; Bystricky et al., 2005). In metaphase, kinetochores did not generate a metaphase plate but rather organized into two sister kinetochore clusters. In late mitosis, the separation of the sister kinetochore clusters increases (Figure 1B) (Joglekar et al., 2006). At this late stage of division, their high density did not allow us to resolve individual kinetochores with SMLM. In conclusion, SMLM allows visualizing single kinetochores within the budding yeast spindle in interphase and in metaphase. 
bioRxiv preprint doi: https://doi.org/10.1101/2021.12.01.469648; this version posted December 3, 2021. The copyright holder for this preprint (which was not certified by peer review) is the author/funder, who has granted bioRxiv a license to display the preprint in perpetuity. It is made available under aCC-BY-NC 4.0 International license.

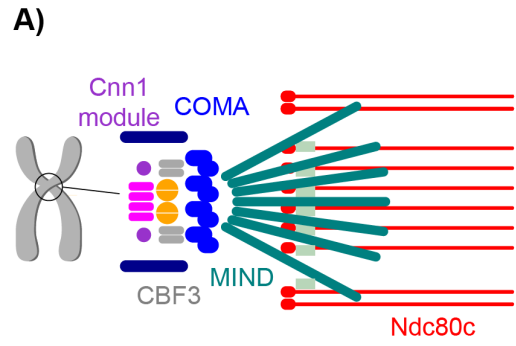

B)
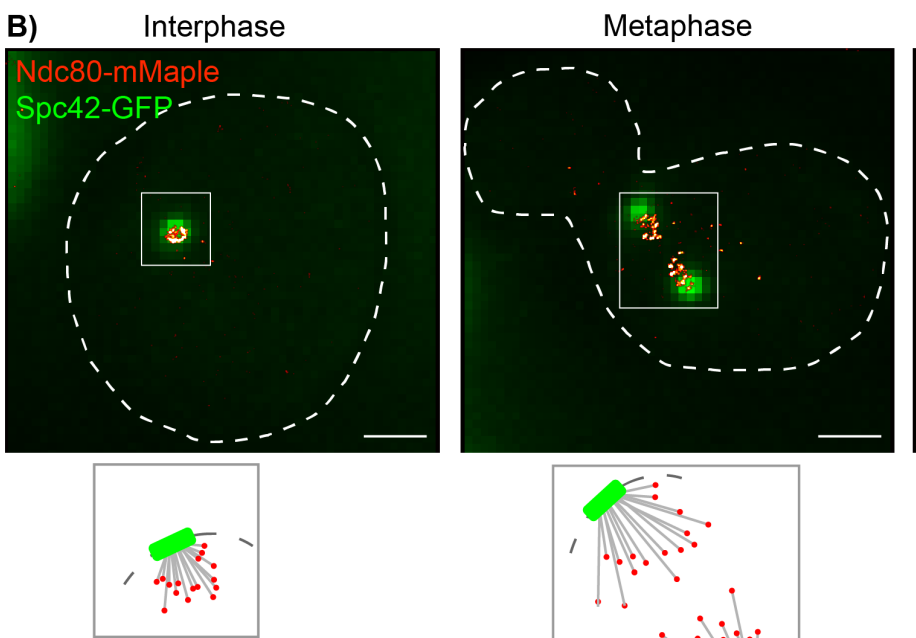

C) Position
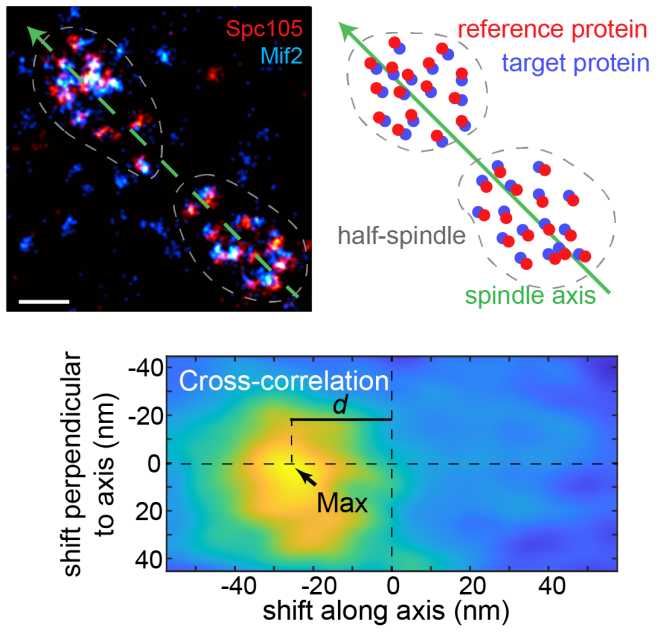

Shift along axis $\rightarrow$ intrakinetochre distance
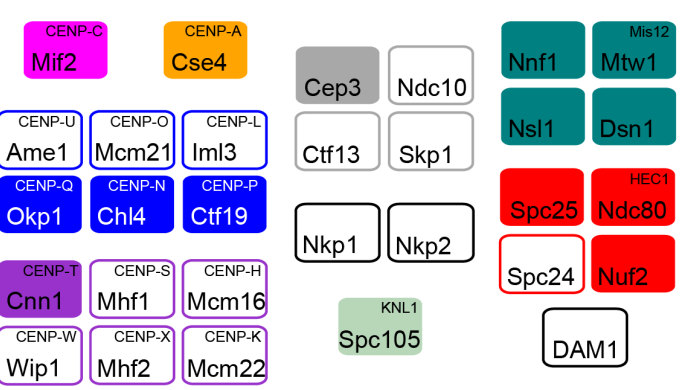

Anaphase
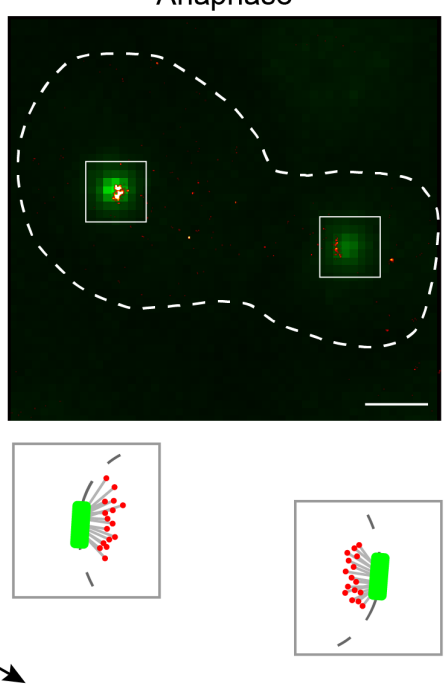

D) Stoichiometry

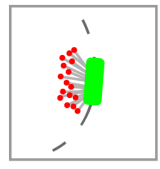

(NPC)
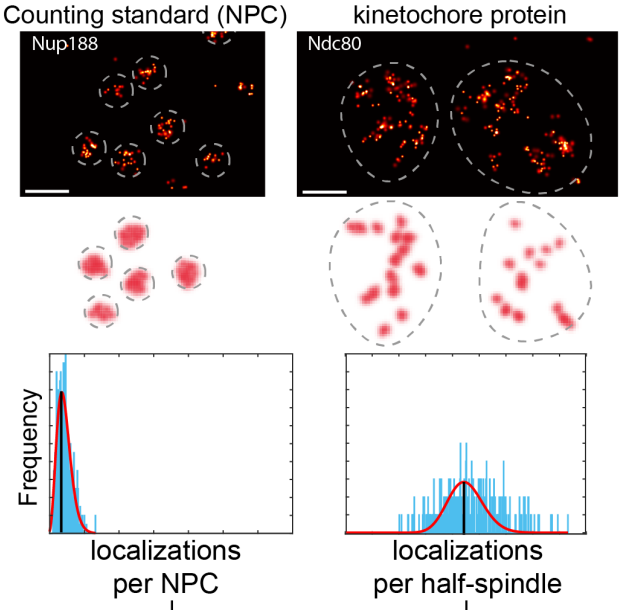

Kinetochore protein copy number

Figure 1: Overview of the study. A. Protein composition of the budding yeast kinetochore. Kinetochore proteins are grouped and color-coded by subcomplexes. Only opaquely colored components were measured in this study. Human counterparts are shown in a superscript. B. Example kinetochore clusters. Representative super-resolved spindle images (red) of the kinetochore protein Ndc80 and the diffraction-limited spindle pole body protein Spc42 (green) at different stages of mitosis phase in the cell cycle and corresponding cartoons of the budding yeast spindles. Scale bars: $1 \mu \mathrm{m}$. $\boldsymbol{C}$. Quantitative SMLM determines the position of kinetochore proteins along the spindle axis. We labeled and imaged always the reference protein Spc105 (red) together with the target protein (cyan, Mif2 in this example). We manually segmented single half-spindles, defined the spindle axis and calculated the image cross-correlation. The position of the cross-correlation peak corresponds to the average distance between reference and target proteins in the half spindle. $\boldsymbol{D}$. Stoichiometry of the budding yeast kinetochore. We quantified the copy numbers of kinetochore proteins using the nuclear pore complex (NPC) component Nup188, which has 16 copies per NPC, as a counting reference standard. In each experiment, we mixed two strains in which either Nup188 or the target kinetochore protein were labeled with the same fluorescence protein tag mMaple. The reference Nup188 strain additionally contained Abp1-GFP for identification. We then imaged both strains under the same conditions at the same time on the same coverslip. We calculated the ratio of mean localization counts per structural unit (either NPC or half-spindle) between the two proteins. From the relative number of localizations and the known stoichiometry of Nup188, we computed the copy number of the target kinetochore protein. Scale bars: $200 \mathrm{~nm}$. 


\section{Dual-color SMLM quantifies positions of kinetochore proteins along the metaphase spindle axis}

In order to resolve structural details of individual kinetochore complexes, we used dual-color superresolution imaging of two kinetochore proteins. We focused on essential kinetochore components and included proteins that have been mapped with diffraction-limited microscopy (Joglekar et al., 2009), for which we could improve the positioning accuracy, and proteins that have never been visualized previously. Unless indicated otherwise, we used Spc105, labeled with SNAP-tag and the organic dye AF647, as a super-resolved spatial reference to position all other proteins, labeled with mMaple, on the spindle axis. To this end, we analyzed each kinetochore cluster individually by reconstructing superresolution images for the reference and target protein and by determining the relative shifts by image cross-correlation (Figure 1C, Supplementary Figure S2, Methods). We only analyzed metaphase cells where both kinetochore clusters allowed for high-quality position measurements. As the two kinetochore clusters have an opposite orientation on the spindle axis, minor registration inaccuracies between the channels share the same amount but opposite signs, therefore cancelling themselves out (Supplementary Figure S2D). This allowed us to determine the pairwise distances between 15 pairs of kinetochore proteins, all labeled at their C-terminus. We further validated this approach with an independent analysis, in which we directly measured the distance of the proteins in individual kinetochores (Figure S1) and obtained highly similar results. Our measurements of different kinetochores are internally consistent, as the sum of the measured Ndc80 - Spc105 (11.5 \pm $1.0 \mathrm{~nm})$ and Spc105 - Ctf19 $(17.7 \pm 1.3 \mathrm{~nm})$ distances is almost identical to the measured Ndc80 Ctf19 distance $(28.5 \pm 1.3 \mathrm{~nm})$ (Figure 2 inset). These data agree reasonably well with previous diffraction-limited dual-color microscopy studies (Joglekar et al., 2009). Furthermore, we found that the C-termini of Ndc80 and Nuf2 are in close proximity with a distance of $3.6 \mathrm{~nm} \pm 1.6 \mathrm{~nm}$ (SEM) (Figure 2), which agrees well with a distance of $3.6 \mathrm{~nm}$, as determined from a crystal structure (Valverde et al., 2016), adding another validation. In summary, these data show that SMLM dualcolor imaging is suitable to measure intra-kinetochore distances in budding yeast.

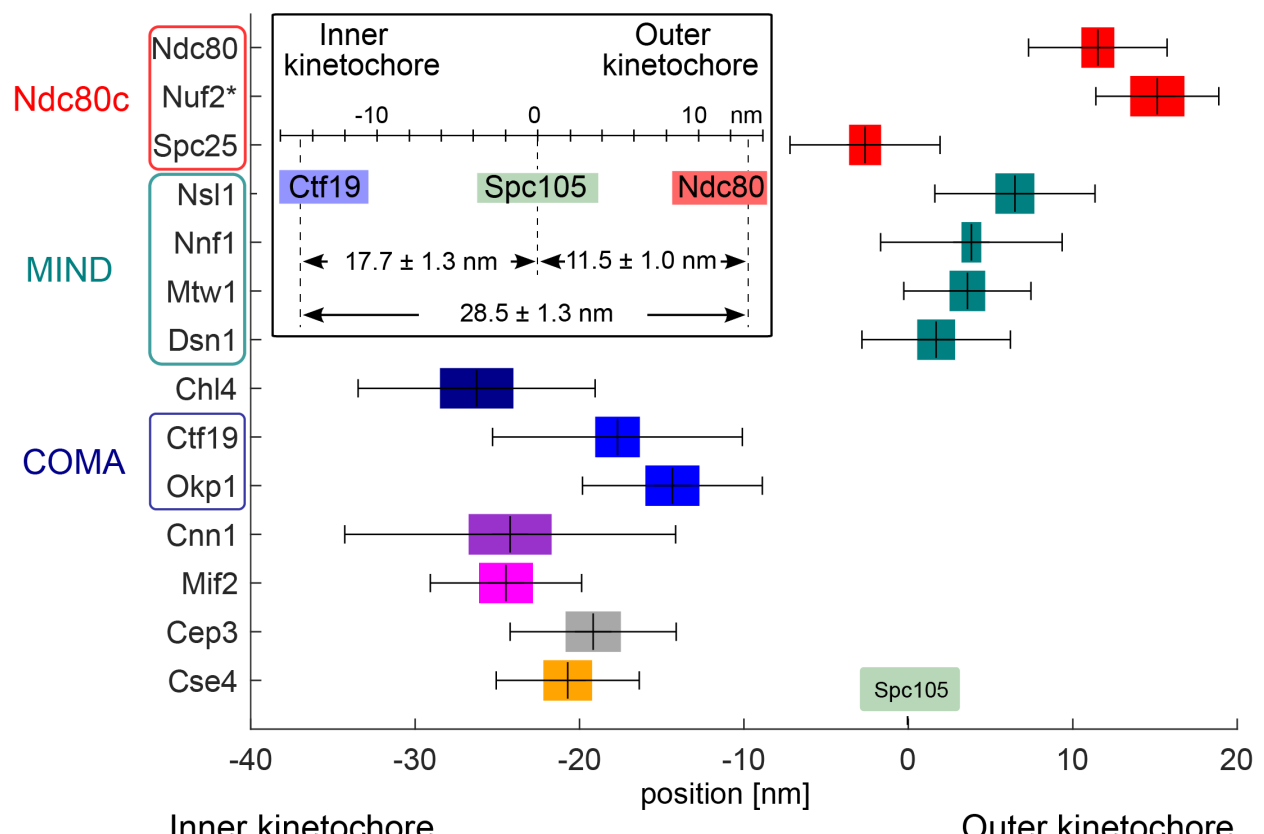

Figure 2. Position of kinetochore proteins along the spindle axis of 14 kinetochore proteins with Spc105 as a reference point. All proteins were tagged at their $C$-termini. The mean distance is plotted with the standard error of mean (SEM, colored box) and standard deviations (STD, whiskers). The inset depicts control measurements showing consistency in pairwise distance measurements \pm SEM among three proteins. See Table 1 for values. 
We found the C-termini of Cse4 and Cep3 to be positioned within $1.7 \mathrm{~nm}$ away from each other at the centromeric site. Also, Mif2 and Cnn1 cluster together, which is consistent with their function within the inner kinetochore (Figure 2) but are $4 \mathrm{~nm}$ away from the Cse4, Cep3 site. Interestingly, we measured the position of Chl4 to be only $1.8 \mathrm{~nm}$ away from Mif2, but more distant from the COMA complex $(8.6 \mathrm{~nm})$. We find that Okp1 and Ctf19 (COMA components) are $19.5 \pm 1.6 \mathrm{~nm}$ and $14.4 \pm$ $1.6 \mathrm{~nm}$ away from Spc105 respectively, towards the centromere (Figure 2).

Finally, we find that Nnf1, Nsl1, Mtw1 and Dsn1, which all belong to the MIND complex are between $1 \mathrm{~nm}$ and $6 \mathrm{~nm}$ away from Spc105 in the outward direction (towards the microtubule). This is consistent with a crystal structure of MIND in yeast and humans and with the known binding site of the KNL1 ${ }^{\text {Spc105 }}$ C-terminus on the MIND complex (Dimitrova et al., 2016; Hornung et al., 2014; Kudalkar et al., 2015; Petrovic et al., 2016; Petrovic et al. 2014). While the C-terminus of Spc25 is adjacent to the C-termini of both Spc105 and MIND (Figure 2), the C-terminus of Ndc80 occupies a more outward position.

\begin{tabular}{ccccc}
\hline Protein & $\begin{array}{c}\text { Distance to } \\
\text { Spc105 (nm) }\end{array}$ & STD & SEM & N \\
\hline Ndc80 & 11.5 & 6.8 & 1.0 & 50 \\
Nuf2* & 15.1 & 6.0 & 1.6 & 14 \\
Spc25 & -2.6 & 4.7 & 0.9 & 26 \\
Ns11 & 6.5 & 5.4 & 1.1 & 22 \\
Nnf1 & 3.8 & 2.8 & 0.6 & 26 \\
Mtw1 & 3.6 & 6.2 & 1.0 & 36 \\
Dsn1 & 1.7 & 6.3 & 1.1 & 32 \\
Ch14 & -26.3 & 12.8 & 2.2 & 34 \\
Ctf19 & -17.7 & 7.9 & 1.3 & 36 \\
Okp1 & -14.4 & 7.8 & 1.6 & 24 \\
Cnn1 & -24.2 & 10.5 & 2.5 & 18 \\
Mif2 & -24.5 & 7.5 & 1.6 & 22 \\
Cep3 & -19.2 & 7.3 & 1.6 & 20 \\
Cse4 & -20.7 & 6.4 & 1.4 & 20 \\
Ctf19-Ndc80 & -28.5 & 10.8 & 1.3 & 68 \\
\hline
\end{tabular}

Table 1. Statistics of kinetochore protein positions along the spindle axis. ${ }^{*}$ The position of Nuf2 is based on the measured pair Ndc80-Nuf2. STD: standard deviation, SEM: standard error of the mean, N: number of spindles.

\section{Counting kinetochore protein copy numbers with quantitative SMLM}

In order to estimate the protein copy numbers of the major kinetochore components, we used a quantitation approach based on super-resolution counting reference standards (Thevathasan et al., 2019). Here, the target complex is imaged under identical conditions as the reference standard, tagged with the same fluorophore (mMaple). The copy number of the unknown complex can be directly calculated from the known copy number of the counting standard and the relative number of detected localizations. We selected Nup188, a protein component of the nuclear pore complex (NPC), as a bright and easy to segment counting reference complex (Figure 1C) (Thevathasan et al., 2019). Nup188 has 16 copies per budding yeast NPC (Kim et al., 2018). We mixed the reference strains containing Nup188-mMaple and Abp1-GFP as an identification marker with the target strains containing mMaple labeled kinetochore proteins and imaged them on the same coverslip to ensure identical imaging conditions. We further improved the accuracy by employing highly homogenous illumination (Deschamps et al., 2016) throughout the entire field of view. We usually acquired images 


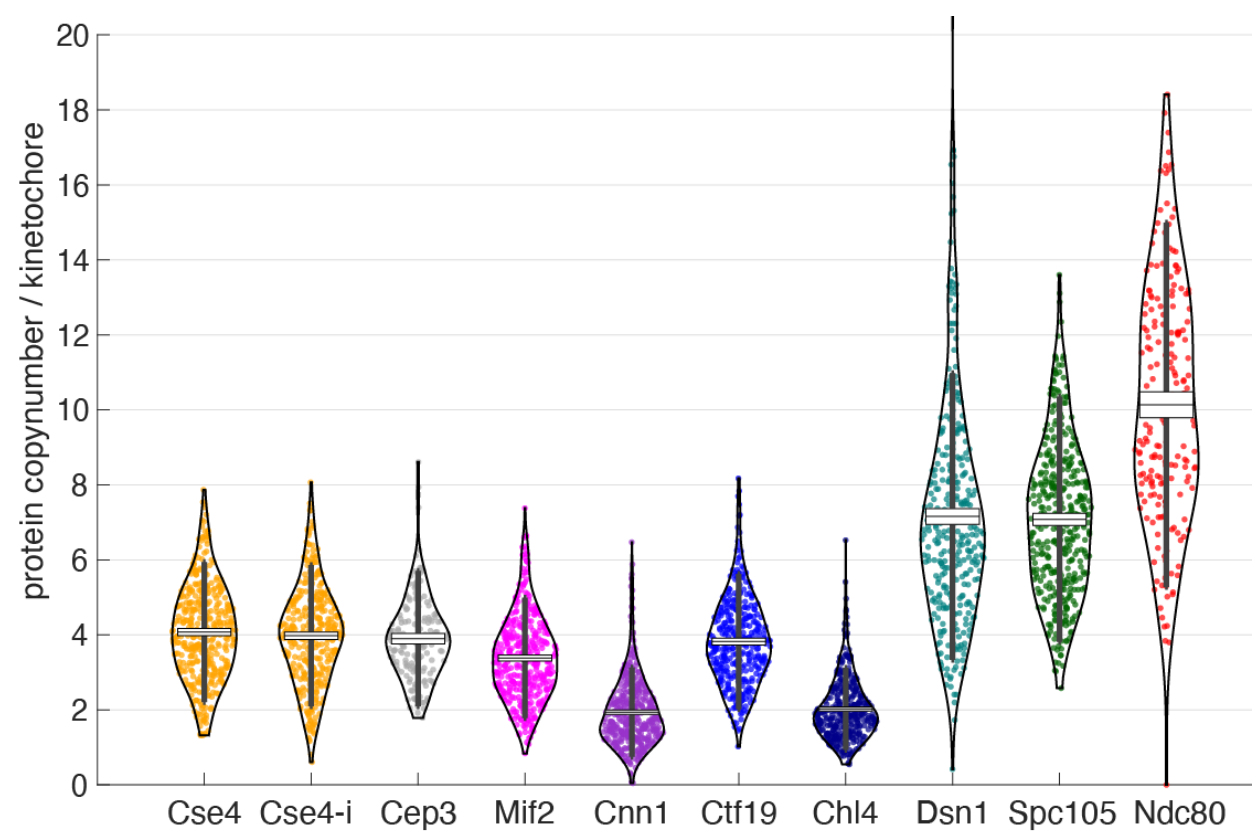

Figure 3. Protein copy numbers per kinetochore measured with Nup188-mMaple as a counting reference standard. Each data point corresponds to one kinetochore cluster. All proteins were tagged at their C-termini, except Cse4-i that was tagged internally. Boxes denote average copy numbers and standard error of means, and whiskers denote standard deviations. For each protein, two independent experiments were performed and pooled (see Methods for details).

of 600 NPCs and 200 kinetochore half-spindles per experiment, which allowed us to precisely calculate the copy numbers of kinetochore proteins.

For the inner kinetochore, we first quantified Cse4. Previous reports have indicated that only internal genetically encoded fluorescent tagging of Cse4 is compatible with its physiological function, while $\mathrm{N}$ - or C-terminal tagging renders cells less viable (Wisniewski et al., 2014). However, in our experiments, we found that both internal and C-terminal tagging of Cse4 were compatible with viability. Furthermore, our counts were essentially identical, with $4.1 \pm 1.9$ (STD) copies of the histone Cse4 when it is tagged internally and $4.0 \pm 1.9$ (Figure 3) if the tag is localized at its Cterminus. Cep3 was found in $3.9 \pm 1.8$ copies. Mif2 and Cnn1 are present in $3.4 \pm 1.6$ and $1.9 \pm 1.2$ copies/kinetochore, respectively. The COMA complex component Ctf19 has $3.8 \pm 1.8$ copies, and the COMA and Mif2 binder Chl4 is present in $2.0 \pm 1.1$ copies. The outer kinetochore proteins are present in higher copy numbers: $7.1 \pm 3.3$ copies of Spc105, $7.2 \pm 3.8$ of Dsn 1 and $10.1 \pm 4.9$ of Ndc80 (Figure 3).

Different life times of the Nup188 and kinetochore proteins could lead to different maturation efficiencies of the mMaple tag and consequently to systematic errors in the counting measurements. To investigate the effect of tag maturation, we transiently stopped all protein translation with 250 $\mathrm{ug} / \mathrm{ml}$ cycloheximide (CHX) and performed our counting measurements $2 \mathrm{~h}$ after this treatment (Figure S3). Although we observed minor changes in copy numbers, the overall effect of CHX was small. We conclude that tag maturation does not affect our measurements of protein copy number. We have not noticed any growth defects that may have arisen from the tagging in our experiments. We do not exclude a possibility of minor effects. However, our data is consistent with the previous measurements suggesting that our C-terminal tagging did not introduced any artefacts (Joglekar et al., 2006, 2008, 2009, Dhatchinamoorthy et al., 2017; Pekgöz Altunkaya et al., 2016, Lawrimore et al. 2011). 


\section{Quantitative model of the budding yeast kinetochore}

We then integrated all protein copy numbers (Figure 3) and distance measurements (Figure 2) in a model of the structural organization of the budding yeast kinetochore (Figure 4). Based on their close proximity (Figure 2) and their known tendency to dimerize (Cohen et al., 2008) we positioned at the centromeric site four copies of Cse4, two CBF3 subunits (with a Cep3 dimer each), two Mif2 dimers and two copies of Cnn1. Between the centromeric site and the outer kinetochore, we positioned four COMA (with four Ctf19) and two Chl4/Iml3. We placed all c-termini of MIND away from COMA. We then positioned seven copies of Spc105 and of MIND and ten globular Spc25-containing ends of NDC80 in close proximity to each other. Four unbound NDC80 were left for Cnn1 binding.
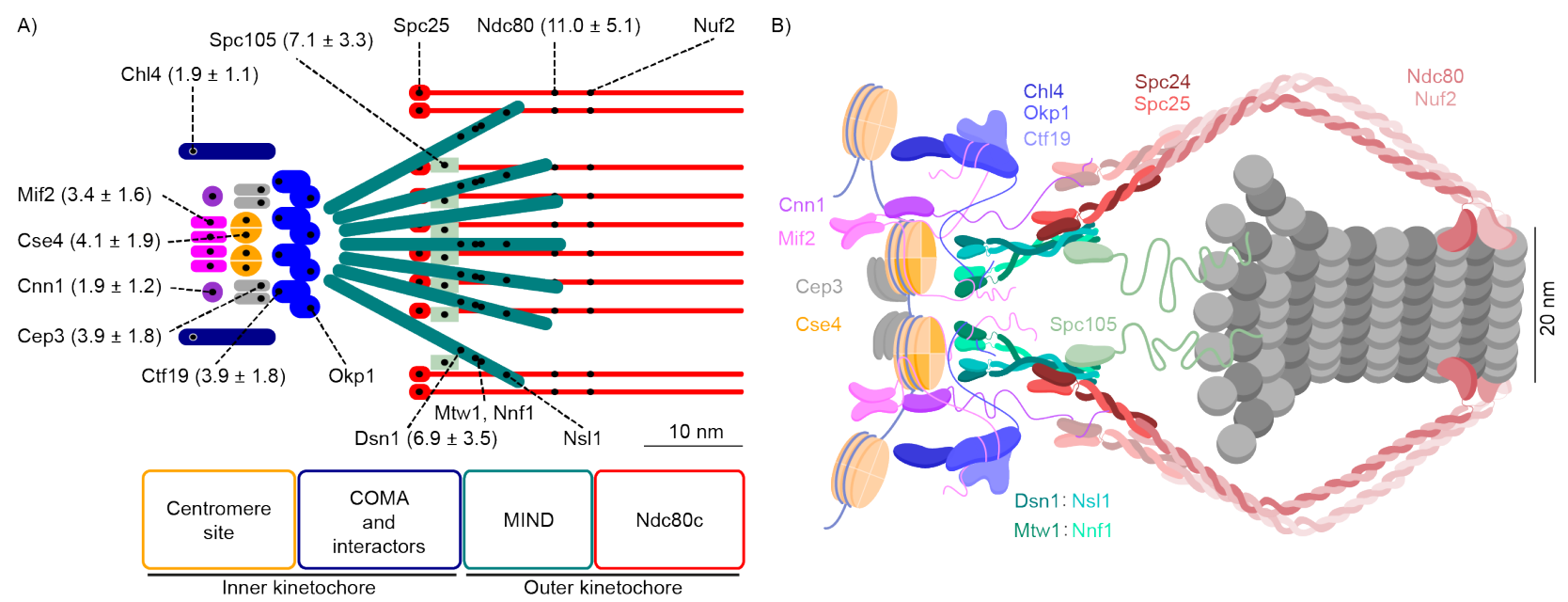

Figure 4. Structural model of the budding yeast kinetochore. A. Quantitative schematic model based on the position and protein copy numbers measured with SMLM. The position of the label is shown as a small black dot. Values in the brackets are the estimates of the number of proteins per kinetochore $+/-$ STD. B. Illustrative structural model that we built by integrating our position and copy number measurements with previous models (Fischböck-Halwachs et al., 2019; Hamilton et al., 2019; Jenni et al., 2017; Ustinov et al., 2020).

\section{Discussion}

In this study, we used single-molecule localization microscopy to position 14 kinetochore proteins along the spindle axis in metaphase and measured the copy numbers of 9 representative kinetochore components (Figure 4), giving new insights into the structural organization of the budding yeast kinetochore in vivo.

\section{Kinetochore subunits are organized functionally along the spindle axis}

Using dual-color SMLM, we mapped the relative positions of 15 kinetochore proteins along the kinetochore axis with nanometer precision. The resulting position map clearly showed that the structural organization of kinetochore proteins correlated with their function and confirmed the general structure of the inner and the outer kinetochore. Kinetochore proteins known to interact with each other were found in close proximity in our analysis, validating the interaction and our approach.

Within the centromere-proximal region, which is more than $20 \mathrm{~nm}$ away from the outer kinetochore and the reference protein Spc105, Cse4 and CBF3 colocalize (measured with its constituent Cep3) with each other as well as with the C-termini of both outer kinetochore receptors Mif2 and Cnn1. The Cep3 dimer, within the CBF3 subcomplex, binds CDEIII DNA and participates in facilitating Cse4containing centromere deposition (Hinshaw and Harrison, 2019; Leber et al., 2018; Yan et al., 2018; 
Zhang et al., 2018). Cnn1 does not seem to bind the centromeric nucleosome directly but its localization depends on Mif2 (Schmitzberger et al., 2017). These generate the base for further kinetochore assembly. Additionally, we find Chl4 within the centromere-proximal region as well, which is in line with Chl4 interacting with Mif2, the Cse4-containing nucleosome and, electrostatically, with DNA (McKinley et al., 2015; Pentakota et al., 2017). The COMA subcomplex (as measured with Ctf19 and Okp1) occupies the intermediate position, 15 to $20 \mathrm{~nm}$ from Spc105, bridging the inner with the outer kinetochore (Hinshaw and Harrison, 2019; Hornung et al., 2014). The outer kinetochore components (Spc105, MIND, NDC80) are the most distal from the centromere and create the microtubule-interacting module, with the NDC80 subcomplex directly binding the microtubule surface (Cheeseman et al., 2006; Ciferri et al., 2008; Wei et al., 2007). All C-termini of the MIND subcomplex are localized more than $10 \mathrm{~nm}$ away from COMA, suggesting that all $\mathrm{N}$ terminal regions of MIND proteins lie relatively close to the subcomplex. This is supported by numerous previous biochemical and optical studies (Aravamudhan et al., 2014; Dimitrova et al., 2016; Petrovic et al., 2016). The distance between the position of COMA and the C-termini of MIND implies a possible tilt between the longer axis of MIND and spindle as the total length of MTW1 is around $20 \mathrm{~nm}$ (Hornung et al., 2011). The structured segment of Spc105, the reference point, is positioned close to the c-termini of MIND, as was proposed previously using structural approaches (Petrovic et al., 2014). The NDC80 subcomplex is an elongated heterotetramer. The C-termini of two of its constituents (Spc25 and Ndc80) are $14.1 \mathrm{~nm}$ away from each other, a few nanometers less than the maximum length of this region observed in the purified sample (Valverde et al., 2016; Wei et al., 2005). Therefore, it is possible that the long axis of NDC80 does not fully align with the kinetochore axis.

Generally, our results align with previous biochemical complex reconstitutions, protein interaction studies and with the majority of optics-based distance measurements. Compared to previous optical measurements, the tenfold higher resolution in our study greatly improved the accuracy of position estimates and the single-kinetochore resolution removed a bias from proteins that are not incorporated in kinetochores but that are nonspecifically enriched in the spindle region. Thus, we found the C-termini of the MIND subcomplex within the outer kinetochore region between Spc105 and the NDC80 complex. Here, c-terminus of Dsn1 highly overlap with Spc105 position, whereas Nnf, Mtw1, Nsl1 c-termini extend towards the position of Ndc80. This adjusts a previous study that measured the distance between the diffraction limited spots of fluorescently tagged kinetochore proteins in living cells and found the C-termini of Mtw1, Ns11 and Dsn1 $7 \mathrm{~nm}$ away from Spc105 in the direction of the centromere, whereas Nnf1 was shown to fully colocalize with Spc105 (Joglekar et al., 2009).

Our results on COMA and NDC80 are compatible with previous studies, but we add position information about important additional proteins that have not been mapped, namely Cse4-C, CBF3, Mif2, Cnn1 and Chl4.

\section{The metaphase kinetochore contains four copies of Cse4}

The quantitative SMLM counting approach recently developed in our lab allowed us to precisely measure the copy number of specific proteins per kinetochore. One highly debated question in the field is the composition of the centromeric nucleosome and, with this, the copy number of Cse4 within individual kinetochores. There is a strong disagreement between biochemical and in-situ assays. Using chromatin immunoprecipitation (ChiP), only a single centromere-specific nucleosome can be recovered (2 Cse4 copies) (Furuyama and Biggins, 2007; Krassovsky et al., 2012). Whereas, 
microscopy data point to higher copy numbers of Cse4, exceeding the expected single centromerespecific nucleosome per kinetochore (Lawrimore et al., 2011). ChiP methods may not be able to detect the additional Cse4 due to their limit of detection (Lawrimore et al., 2011).

In our study, we find four copies of Cse4 per kinetochore, independently of whether tagging was internal (near the N-terminus) or at the C-terminus (Wisniewski et al., 2014). To obtain further information about the centromere environment, we measured the copy numbers of the Cse4-binders Mif2 and Cep3 (CBF3 subcomplex). Consistently, we found that Cse4, Mif2 and Cep3 all have an equal copy number of 4 per kinetochore. A dimer of the CBF3 subcomplex containing two Cep3 dimers was shown to allow integration of a single or two centromere-specific nucleosomes (Yan et al., 2018). Thus, we propose that the CBF3 dimer (with two Cep3 homodimers) accommodates two nucleosome octamers, each containing one Cse4 dimer. In turn, this suggests that each nucleosome may form a complex with the Mif2 dimer, in line with a recent report (Xiao et al., 2017). It is worth noting that in other organisms the Cenp-C dimer may interact with two centromeric nucleosomes distinguishing the budding yeast centromere even more (Carroll et al., 2010; Guse et al., 2011; Watanabe et al., 2019; Ali-Ahmad et al., 2019; Walstein et al., 2020). Taken together, our data supports a model where a CBF3 dimer allows incorporation of two octameric nucleosomes. The arrangement may generate a larger outer kinetochore receptor interface (Klare et al., 2015) by providing four, instead of two, Mif2 N-termini. Alternatively, non-centromeric Cse4 may play a role in maintaining the "point" centromere (as discussed in Scott and Bloom, 2014) by serving as a spare module.

\section{Copy numbers of the major kinetochore components}

In our study, we found four copies of Ctf19 but only two copies of Chl4 per kinetochore, indicating that a kinetochore contains four COMA subcomplexes and two Chl4/Iml3 subcomplexes. This is consistent with the interaction of one CENP-L/N dimer to one CENP-A nucleosome in human cells (Pentakota et al., 2017), and suggests Chl4/Iml3 binds to the Cse4 nucleosome with a 1:1 stoichiometry. It is widely accepted that N-termini of both Mif2 protein and COMA subunits allow and regulate assembly of the outer kinetochore module (Dimitrova et al., 2016; Petrovic et al., 2016; Przewloka et al., 2011; Screpanti et al., 2011). With a total of eight interaction sites from two Mif2 dimers and four COMA, a budding yeast kinetochore may generate up to eight copies of MIND. This in turn would bring equal amount of Spc105 and Ndc80 subcomplexes (Petrovic et al., 2014). Indeed, we observed 7-8 MIND and an equal number of Spc105. Consistently with others (Joglekar et al., 2006; Dhatchinamoorthy et al., 2017; Joglekar et al., 2006) we found more Ndc80 than Spc105 or MIND per kinetochore. The additional $2-3$ Ndc80 can be bound by the last outer kinetochore receptor Cnn1. In regional kinetochores, Cenp-T, the Cnn1 orthologue, recruits up to three NDC80 to the outer kinetochore (Huis in 't Veld et al., 2016). In budding yeast, each Cnn1 can bind two NDC80 (Pekgöz Altunkaya et al., 2016). The binding is regulated by Cdk1 and Mps1 dependent phosphorylation of Cnn1 (Malvezzi et al., 2013). The decreasing activity of the aforementioned kinases may allow the Cnn1-Ndc80 interaction to be more permissive. Our observations were limited only to metaphase. Therefore, our observation is consistent with one Cnn1 binding to a total of two to three Ndc80 per kinetochore. Yet, when NDC80 copy numbers are estimated in Cnn1 deleted strains the copy number is not altered (Dhatchinamoorthy et al., 2017; Pekgöz Altunkaya et al., 2016) or the change may be minimal when MIND-NDC80 binding pathway is impaired (Lang et al., 2018). This points to the redundancy of Cnn 1 in budding yeast when the mitotic checkpoint is not compromised, or to a dynamic nature of the Ndc80-Cnn1 interaction. 


\section{Summary}

Taken together, we have employed the high resolution of SMLM to substantially improve the accuracy of previous stoichiometry and intra-kinetochore distance estimates and obtained a comprehensive model of the structural organization of the kinetochore in budding yeast in situ (Figure 4), revising previous models (Jenni et al., 2017; Fischböck-Halwachs et al., 2019; Hamilton et al., 2019; Ustinov et al., 2020). This model adds additional valuable information to understand how the metaphase kinetochore is structurally organized in situ by overcoming the resolution limit present in the previous studies. Specifically, we proposed a possible alternative model in which the inner kinetochore may be based on two sets of centromeric nucleosomes and CBF3, instead of one. Both Cse4-containing nucleosomes may be involved in determining the structure of the microtubule-binding module by interacting with two Mif2 dimers. In our model additional Cse4 are not accessory but are an integrative part of the budding yeast kinetochore.

In an independent investigation, a similar methodology was used to assess protein composition and distances of $S$. pombe kinetochores (Virant et al., 2021). Their results are in excellent agreement with ours, as expected from the high conservation of kinetochore components across the two yeast strains (Van Hoof et al., 2017), validating our respective approaches. One main difference is the Cse4:COMA ratio, which is 1:0.9 in buddying yeast and 1:2.1 in fission yeast, pointing to intrinsic stoichiometry changes between point and regional kinetochores. In conclusions, our quantitative SMLM methods provide an excellent basis for future studies, for instance how kinetochore components are organized perpendicular to the spindle axis and how this relates to the kinetochore-microtubule binding management, how structure and stoichiometry change throughout the cell cycle or how kinetochores are organized in other organisms. Our methods are not restricted to kinetochores, but will enable quantitative measurements of the stoichiometry and structure of other multi-protein assemblies in situ.

\section{Acknowledgements}

We thank Andrea Musacchio and Ulrike Endesfelder for feedback on the manuscript and Katharina Lindner for her work on the imaging of the dual color strains. This work was supported by the European Research Council (grant no. ERC CoG-724489 to JR), the Human Frontier Science Program (RGY0065/2017 to JR) and the European Molecular Biology Laboratory.

\section{Methods}

\section{Yeast strain generation}

All strains used in the study (Table S1) were derived from $S$. cerevisiae MKY0100 and MKY1743 (for $\mathrm{N}$-terminal cloning), a kind gift from the Kaksonen lab. The strains were created by homologues recombination using PCR-based carboxy-terminal (C-terminal) tagging (Janke et al., 2004). The tagging cassettes are created by amplification of DNA regions in pFA6a plasmids (Mund et al., 2018) encoding mMaple (McEvoy et al., 2012) or SNAPf-tag (Sun et al., 2011). Each primer consists of a 5' overhang that encodes a $\sim 50$-base-long homologous sequence to the gene of interest and a 3 ' region that anneals to the plasmid used in the PCR. The Cse4-mMaple-Cse4 strain was created analogically to (Wisniewski et al., 2014). Cse4 and mMaple sequences were amplified by PCR and ligated into pFA6a vector replacing a tag sequence. Subsequently PCR product encoding Cse4-mMaple-Cse4HIS3MX6 was used to transform competent cells. 
The competent cells were prepared by restreaking a single colony onto a YPAD plate and letting them grow for approx. 3 days. Then, $4 \mathrm{ml}$ of YPAD were inoculated with a freshly grown colony. The cells were cultured by shaking $\left(220 \mathrm{rpm}, 30^{\circ} \mathrm{C}\right)$ overnight. The cells were diluted to $0.2 \mathrm{OD}$ in $50 \mathrm{ml}$ of fresh YPAD and cultured until mid-log phase. Subsequently, the cells were washed twice by spinning down (1500 rpm, $5 \mathrm{~min}$. at room temperature). After the first wash the cells were resuspended in sterile water then with SORB buffer. In the meantime, $40 \mu \mathrm{l}$ of $2 \mathrm{mg} / \mathrm{ml}$ salmon sperm DNA were denatured at $100^{\circ} \mathrm{C}$ for $10 \mathrm{~min}$. then cooled on ice. Next, the cells pellet was resuspended in $360 \mu \mathrm{l}$ of SORB buffer and $40 \mu \mathrm{l}$ of the salmon sperm DNA. The cells were aliquoted into $\sim 10$ tubes with 50 $\mu \mathrm{l}$ each and stored at $-80^{\circ} \mathrm{C}$.

For the transformation reaction, $15 \mu \mathrm{l}$ of unpurified PCR product were gently mixed with $50 \mu \mathrm{l}$ of the competent cells, $360 \mu \mathrm{l}$ of PEG buffer and left at room temperature for $30 \mathrm{~min} .47 \mu \mathrm{l}$ of DMSO were added to the reaction followed by $15 \mathrm{~min}$. incubation at $42^{\circ} \mathrm{C}$. After the heat-shock the cells were spun down at $2100 \mathrm{rpm}$ for 2,5 min. The pellet was resuspended in water and plated onto a selection plate. The plate was then incubated at $30^{\circ} \mathrm{C}$ for 3 days. Fully grown colonies were checked by diagnostic PCR.

\section{Sample preparation}

$24 \mathrm{~mm}$ round coverslips were cleaned in $\mathrm{HCl} / \mathrm{Methanol}$ overnight and then rinsed with water. Additionally, the coverslips were cleaned using a plasma cleaner to remove residual organic contaminations. In the meantime, $4 \mathrm{mg} / \mathrm{ml}$ Concanavalin A solution (in PBS) was spun down (14000 rcf, 5 min.). Each coverslip was then coated with $15 \mu$ l of Concanavalin A. Subsequently, the coverslips were incubated for $30 \mathrm{~min}$. at room temperature in moist conditions. Finally, the residual liquid was pipetted out from the coverslips' surface and dried overnight at $37^{\circ} \mathrm{C}$. Before use, the coverslips were rinsed with water to remove salts from PBS. Then, the coverslip was covered with $\sim 100 \mu$ l of a cell suspension and incubated for $15 \mathrm{~min}$.

For mMaple imaging, a single colony was restreaked onto a fresh YPAD plate and incubated at $30^{\circ} \mathrm{C}$ for 2-3 days. Then, $4 \mathrm{ml}$ of SC-Trp, inoculated with a colony, was shaken $(220 \mathrm{rpm})$ overnight. In the morning, the cells were diluted to $0.25 \mathrm{OD}$ in $10 \mathrm{ml}$ of SC-Trp and cultured until the logarithmic phase ( $\sim 3 \mathrm{~h}$ ). $2 \mathrm{ml}$ of the cells were spun down (2500 rpm, $3 \mathrm{~min}$.) and resuspended in $100 \mu \mathrm{l}$ of the medium. In case of the control experiments with cycloheximide treatment, $250 \mu \mathrm{g} / \mathrm{ml}$ of cycloheximide (in DMSO) was added to cells for $1 \mathrm{hr}$ before immobilization. The cells were immobilized on Concanavalin A-coated coverslips. Subsequently, the sample was fixed in $4 \%$ paraformaldehyde-based fixation buffer for $15 \mathrm{~min}$. at room temperature and quenched in $100 \mathrm{mM}$ ammonium chloride pH 7,5 in PBS for 20 min. twice. Finally, the sample was rinsed with PBS several times. The coverslip was mounted on a microscope stage and covered with $50 \mathrm{mM}$ Tris/ $\mathrm{HCl} \mathrm{pH} 8$ in $95 \% \mathrm{D}_{2} \mathrm{O}$.

For single- and dual-color imaging with SNAP, the cells were immobilized, fixed and washed the same way as in the previous paragraph. Subsequently, the sample was covered with $0.01 \%$ digitonin in $1 \%$ BSA solution and incubated for $30 \mathrm{~min}$. at room temperature under moist conditions. The sample was then washed in PBS. The sample was labeled with $1 \mu \mathrm{M}$ SNAP-Surface Alexa Fluor 647 in 1\% BSA solution for $2 \mathrm{~h}$ at room temperature under moist conditions. Finally, the sample was washed in PBS $3 \times 5$ min. The sample was mounted in a microscope stage and covered with the blinking buffer 50 $\mathrm{mM}$ Tris/HCl pH 8, $10 \mathrm{mM} \mathrm{NaCl}, 10 \%$ (w/v) D-glucose, $500 \mu \mathrm{g} / \mathrm{ml}$ Glucose oxidase, $40 \mu \mathrm{g} / \mathrm{ml}$ Glucose catalase in $90 \% \mathrm{D}_{2} 0$ (Thevathasan et al. 2019). The blinking buffer for single-color or dualcolor imaging was supplemented with $35 \mathrm{mM}$ or $15 \mathrm{mM}$ MEA respectively. 


\section{Microscopy}

The SMLM acquisitions were performed with the two custom-build microscopes, analogically as in (Mund et al., 2018) and with custom-developed EMU interface (Deschamps and Ries, 2020). Microscope 1 was used for low-throughput single- and dual-color imaging. Before the dual-color experiment, a bead calibration for a faithful channel overlay was performed. For this, $2.5 \mu 1$ of vortexed $100 \mathrm{~nm}$ Tetraspeck beads were diluted with $400 \mu \mathrm{l}$ of $10 \mathrm{mM} \mathrm{MgCl}_{2}$. The beads were allowed to settle down on a coverslip. Then, the position of the tube lens of the second channel was adjusted to focus both channels in the same focal place. The splitting of the emission signals was achieved with a $640 \mathrm{~nm}$ long pass dichroic mirror. The signal from the $640 \mathrm{~nm}$ or $562 \mathrm{~nm}$ excitation was collected through $676 / 37 \mathrm{~nm}$ or $600 / 60 \mathrm{~nm}$ emission bandpass filters, respectively. 70 images from different parts of the coverslips were collected with $50 \mathrm{~ms}$ exposure time. SMLM measurements were performed with $30 \mathrm{~ms}$ exposure time. The UV laser was adjusted automatically to keep the density of localizations constant (Mud et al. 2018). For dual-color imaging, the sample was mounted in the blinking buffer containing $15 \mathrm{mM}$ of MEA. The cells with similarly bright sister kinetochore signals were chosen for each acquisition. Initially, we imaged the cells with the $640 \mathrm{~nm}$ laser until the localization density was sufficiently reduced. Then the $561 \mathrm{~nm}$ laser was added. Typically, we acquired 60000 frames and obtained $\sim 35 \mathrm{~nm}$ localization precision for mMaple $20 \mathrm{~nm}$ for Alexa Fluor 647.

Microscope 2 was primarily used for high-throughput single-color imaging. As in microscope 1, microscope 2 has several available channels/colors - UV (405 nm), green (488 nm laser, 525/50 nm emission bandpass filter), orange (561 nm laser, 600/60 nm emission bandpass filter), red (640 nm - excitation and booster laser, 700/100 nm emission bandpass filter). A focus lock system based on a totally reflected IR laser beam was used to keep the focus constant. In order to keep the entire field of view uniform we used homogenous and speckle-free free illumination (Deschamps et al. 2016).

High-throughput imaging for counting of mMaple labeled proteins was performed only on Microscope 2. Both strains expressing the Nup188-mMaple brightness standard and the target kinetochore protein labeled with mMaple were mixed and imaged simultaneously. 225 regions were imaged per coverslip, separated by at least $150 \mu \mathrm{m}$ to avoid premature activation. Every acquisition was performed with approximately $100 \mathrm{~mW}$ of the $561 \mathrm{~nm}$ laser, $25 \mathrm{~ms}$ exposure time and the UV laser adjusted automatically to result in a constant, but low density of activated fluorophores. All measurements were performed until all mMaple fluorophores had been activated and bleached. A snapshot of Ndc80-GFP (for kinetochores) or Abp1-GFP (for Nup188-mMaple) was automatically acquired, as well as a back focal plane image to exclude air bubbles.

\section{Single-molecule localization}

We used SMAP (Ries, 2020) for all data analysis. For single-molecule fitting, candidate localizations were detected by smoothing with a Difference of Gaussians filter and thresholding. Then, the signal was localized by fitting a Gaussian function with a homogeneous photon background, treating the size of the Gaussian as a free fitting parameter. Fluorophores spanning consecutive frames and thus likely stemming from the same fluorophore were merged (grouped) into a single localization. For experiments longer than 5000 frames, cross-correlation based sample drift correction was applied as described in (Mund et al., 2018). Super-resolution images were reconstructed by rendering each localization as a Gaussian with a size proportional to the localization precision. Finally, localizations were filtered to exclude poor localization precisions of more than $20 \mathrm{~nm}$ for AlexaFuor647 and 25 $\mathrm{nm}$ for mMaple, and to exclude fitted sizes of the Gaussian of more than $160 \mathrm{~nm}$, which correspond 
to out-of-focus fluorophores. If the localization density in the first frames was above the single molecule regime, these frames were discarded.

Dual-color bead images were fitted as described above and used to calculate a projective transformation between the channels.

For high-throughput data we extracted additional parameters for quality control such as the the number of localizations and the median localization precision, photon count, PSF size and background and used them in combination with the BFP images to exclude poor measurements that resulted from air bubbles in the immersion oil or acidification of the buffer.

\section{Quantification of intrakinetochore distances}

We quantified intrakinetochore distances based on a cross-correlation analysis. In a dual-color SMLM data set, one color/channel (usually the channel of Spc105 unless specified otherwise) was defined as the reference, and the other as the target. We started by manually collecting half-spindles (sites) and grouped both half-spindles of the same mitotic spindle as a pair (Figure S2A). For each pair, a line was manually drawn to represent the spindle axis, which the half-spindles distributed along. Next, to take the opposite direction of chromosomes pulling by each half-spindle of the pair into account, the axial direction was defined as pointing towards the center of the spindle (Figure S2A). As shown in Figure S2B, each half-spindle/spindle went through the same analysis steps (Figure S2C and D) for quantifying the distance. First, we calculated the image cross-correlation between two reconstructed super-resolution images corresponding to the two channels for each half-spindle separately. From the maximum position of the cross-correlation map we determined the average distance between the two proteins along the spindle axis. To exclude that residual transformation errors caused e.g., by chromatic aberrations result in a bias, we always analyzed the two paired halfspindles together. Due to their close proximity, we expect similar registration errors, which cancel out when calculating the average protein distance because of the opposite orientation of the halfspindles. As a result, each spindle resulted in one average distance value. Using Spc105 as a reference in most data sets, we could position all measured proteins along the spindle axis.

\section{Protein copy number estimations}

To differentiate the cell lines in the same specimen, proteins (Abp1 for the reference and a kinetochore protein for a target cell line) with different cellular distributions were tagged with mEGFP in the reference and target cell lines. The GFP signal was checked in the diffraction-limited channel. We then manually segmented the single structures of the reference (NPCs) and the target (half-spindles) in respective cell lines. Only the in-focus structures were picked. For the reference, NPCs at the edge of the nucleus or were too close to neighboring structures were excluded. We then determined the number of localizations in a circular ROI of a diameter of $150 \mathrm{~nm}$. For a target structure, we only picked half-spindles that have two foci in the GFP channel to ensure metaphase half-spindles. We then determined the number of localizations in the manually-created polygon enclosing the halfspindle. When paired half-spindles were too close to each other, they were segmented as one entity and its localizations were divided by 2 . Then the copy number $N_{k}$ of a target protein per kinetochore was calculated as $N_{k}=N_{n}\left(\frac{L_{h s}}{N_{h s}}\right) / L_{n}$, where both $N_{n}$ and $N_{h s}$ are 16, the known protein copy number of NPC and the number of kinetochores per half-spindle respectively. $L_{n}$ and $L_{h s}$ are the mean quantified localizations per NPC and per half-spindle respectively. To take the variation of the NPC localizations into account, the standard deviation of the kinetochore protein copy number was 
estimated as $S=N_{k} \sqrt{\left(S_{n} / \mathrm{L}_{n}\right)^{2}+\left(S_{k} / \mathrm{L}_{k}\right)^{2}}$, where $\mathrm{S}_{k}$ and $\mathrm{S}_{n}$ are the standard deviations of the localizations for NPC and kinetochore protein and $M_{k}$ and $M_{n}$ are the respective sample sizes. Finally, the pooled copy number and standard deviation of replicates were defined as $\overline{N_{k}}=$ $\left.\left(N_{k 1} M_{k 1}+N_{n 2} M_{n 2}\right) /\left(M_{k 1}+M_{k 2}\right)\right) \quad$ and $\quad \bar{S}=\sqrt{\left.\left(\left(M_{k 1}-1\right) S_{1}{ }^{2}+\left(M_{k 2}-1\right) S_{2}{ }^{2}\right) /\left(M_{k 1}+M_{k 2}-2\right)\right)}$ respectively.

\section{References}

Abbe, E., 1873. Beiträge zur Theorie des Mikroskops und der mikroskopischen Wahrnehmung. Archiv für mikroskopische Anatomie 9, 413-418.

Ali-Ahmad, A., Bilokapić, S., Schäfer, I.B., Halić, M., Sekulić, N., 2019. CENP-C unwraps the human CENP-A nucleosome through the H2A C-terminal tail. EMBO reports 20, e48913. https://doi.org/10.15252/embr.201948913

Aravamudhan, P., Felzer-Kim, I., Gurunathan, K., Joglekar, A.P., 2014. Assembling the Protein Architecture of the Budding Yeast Kinetochore-Microtubule Attachment using FRET. Current Biology 24, 1437-1446. https://doi.org/10.1016/j.cub.2014.05.014

Aravamudhan, P., Goldfarb, A.A., Joglekar, A.P., 2015. The kinetochore encodes a mechanical switch to disrupt spindle assembly checkpoint signalling. Nature Cell Biology 17, 868-879. https://doi.org/10.1038/ncb3179

Asbury, C.L., 2017. Anaphase A: Disassembling Microtubules Move Chromosomes toward Spindle Poles. Biology 6, 15. https://doi.org/10.3390/biology6010015

Betzig, E., Patterson, G.H., Sougrat, R., Lindwasser, O.W., Olenych, S., Bonifacino, J.S., Davidson, M.W., Lippincott-Schwartz, J., Hess, H.F., 2006. Imaging Intracellular Fluorescent Proteins at Nanometer Resolution. Science 313, 1642-1645. https://doi.org/10/dd8dkd

Black, B.E., Cleveland, D.W., 2011. Epigenetic Centromere Propagation and the Nature of CENP-A Nucleosomes. Cell 144, 471-479. https://doi.org/10.1016/j.cell.2011.02.002

Bui, M., Dimitriadis, E.K., Hoischen, C., An, E., Quénet, D., Giebe, S., Nita-Lazar, A., Diekmann, S., Dalal, Y., 2012. Cell-Cycle-Dependent Structural Transitions in the Human CENP-A Nucleosome In Vivo. Cell 150, 317-326. https://doi.org/10.1016/j.cell.2012.05.035

Bystricky, K., Laroche, T., van Houwe, G., Blaszczyk, M., Gasser, S.M., 2005. Chromosome looping in yeast telomere pairing and coordinated movement reflect anchoring efficiency and territorial organization. $\mathrm{J}$ Cell Biol 168, 375-387. https://doi.org/10.1083/jcb.200409091

Camahort, R., Shivaraju, M., Mattingly, M., Li, B., Nakanishi, S., Zhu, D., Shilatifard, A., Workman, J.L., Gerton, J.L., 2009. Cse4 Is Part of an Octameric Nucleosome in Budding Yeast. Molecular Cell 35, 794-805. https://doi.org/10.1016/j.molcel.2009.07.022

Carroll, C.W., Milks, K.J., Straight, A.F., 2010. Dual recognition of CENP-A nucleosomes is required for centromere assembly. J Cell Biol 189, 1143-1155. https://doi.org/10.1083/jcb.201001013

Chaly, N., Brown, D.L., 1988. The prometaphase configuration and chromosome order in early mitosis. J Cell Sci 91 ( Pt 3), 325-335.

Cheeseman, I.M., Chappie, J.S., Wilson-Kubalek, E.M., Desai, A., 2006. The Conserved KMN Network Constitutes the Core Microtubule-Binding Site of the Kinetochore. Cell 127, 983-997. https://doi.org/10.1016/j.cell.2006.09.039

Ciferri, C., Pasqualato, S., Screpanti, E., Varetti, G., Santaguida, S., Dos Reis, G., Maiolica, A., Polka, J., De Luca, J.G., De Wulf, P., Salek, M., Rappsilber, J., Moores, C.A., Salmon, E.D., Musacchio, A., 2008. Implications for Kinetochore-Microtubule Attachment from the Structure of an Engineered Ndc80 Complex. Cell 133, 427-439. https://doi.org/10.1016/j.cell.2008.03.020

Clarke, L., Carbon, J., 1980. Isolation of a yeast centromere and construction of functional small circular chromosomes. Nature 287, 504-509. https://doi.org/10.1038/287504a0

Cohen, R.L., Espelin, C.W., De Wulf, P., Sorger, P.K., Harrison, S.C., Simons, K.T., 2008. Structural and Functional Dissection of Mif2p, a Conserved DNA-binding Kinetochore Protein. MBoC 19, 4480-4491. https://doi.org/10.1091/mbc.e08-03-0297

Conti, D., Hart, M., Tamura, N., Shrestha, R., Islam, A., Draviam, V.M., 2017. How are Dynamic Microtubules Stably Tethered to Human Chromosomes? Cytoskeleton - Structure, Dynamics, Function and Disease. https://doi.org/10.5772/intechopen.68321

Dalal, Y., Furuyama, T., Vermaak, D., Henikoff, S., 2007. Structure, dynamics, and evolution of centromeric nucleosomes. Proc Natl Acad Sci USA 104, 15974. https://doi.org/10.1073/pnas.0707648104 
Dani, A., Huang, B., Bergan, J., Dulac, C., Zhuang, X., 2010. Superresolution Imaging of Chemical Synapses in the Brain. Neuron 68, 843-856. https://doi.org/10.1016/j.neuron.2010.11.021

Deschamps, J., Ries, J., 2020. EMU: reconfigurable graphical user interfaces for Micro-Manager (preprint). Bioinformatics. https://doi.org/10.1101/2020.03.18.997494

Deschamps, J., Rowald, A., Ries, J., 2016. Efficient homogeneous illumination and optical sectioning for quantitative single-molecule localization microscopy. Optics express 24, 28080-28090.

Dhatchinamoorthy, K., Shivaraju, M., Lange, J.J., Rubinstein, B., Unruh, J.R., Slaughter, B.D., Gerton, J.L., 2017. Structural plasticity of the living kinetochore. J Cell Biol 216, 3551-3570. https://doi.org/10.1083/jcb.201703152

Dimitrova, Y.N., Jenni, S., Valverde, R., Khin, Y., Harrison, S.C., 2016. Structure of the MIND Complex Defines a Regulatory Focus for Yeast Kinetochore Assembly. Cell 167, 1014-1027.e12. https://doi.org/10.1016/j.cell.2016.10.011

Drinnenberg, I.A., Henikoff, S., Malik, H.S., 2016. Evolutionary Turnover of Kinetochore Proteins: A Ship of Theseus? Trends in Cell Biology 26, 498-510. https://doi.org/10.1016/j.tcb.2016.01.005

Fischböck-Halwachs, J., Singh, S., Potocnjak, M., Hagemann, G., Solis-Mezarino, V., Woike, S., GhodgaonkarSteger, M., Weissmann, F., Gallego, L.D., Rojas, J., Andreani, J., Köhler, A., Herzog, F., 2019. The COMA complex interacts with Cse4 and positions Sli15/Ipl1 at the budding yeast inner kinetochore. eLife 8. https://doi.org/10.7554/eLife.42879

Furuyama, S., Biggins, S., 2007. Centromere identity is specified by a single centromeric nucleosome in budding yeast. PNAS 104, 14706-14711. https://doi.org/10.1073/pnas.0706985104

Gonen, S., Akiyoshi, B., Iadanza, M.G., Shi, D., Duggan, N., Biggins, S., Gonen, T., 2012. The structure of purified kinetochores reveals multiple microtubule-attachment sites. Nature Structural \& Molecular Biology 19, 925-929. https://doi.org/10.1038/nsmb.2358

Guse, A., Carroll, C.W., Moree, B., Fuller, C.J., Straight, A.F., 2011. In vitro centromere and kinetochore assembly on defined chromatin templates. Nature 477, 354-358. https://doi.org/10.1038/nature10379

Haase, J., Mishra, P.K., Stephens, A., Haggerty, R., Quammen, C., Taylor, R.M., Yeh, E., Basrai, M.A., Bloom, K., 2013. A 3D Map of the Yeast Kinetochore Reveals the Presence of Core and Accessory CentromereSpecific Histone. Current Biology 23, 1939-1944. https://doi.org/10.1016/j.cub.2013.07.083

Hamilton, G., Dimitrova, Y., Davis, T.N., 2019. Seeing is believing: our evolving view of kinetochore structure, composition, and assembly. Current Opinion in Cell Biology 60, 44-52. https://doi.org/10.1016/j.ceb.2019.03.016

Hess, S.T., Girirajan, T.P.K., Mason, M.D., 2006. Ultra-High Resolution Imaging by Fluorescence Photoactivation Localization Microscopy. Biophysical Journal 91, 4258-4272. https://doi.org/10/cr8s93

Hinshaw, S.M., Harrison, S.C., 2019. The structure of the Ctf19c/CCAN from budding yeast. eLife 8, e44239. https://doi.org/10.7554/eLife.44239

Hooff, J.J., Tromer, E., Wijk, L.M., Snel, B., Kops, G.J., 2017. Evolutionary dynamics of the kinetochore network in eukaryotes as revealed by comparative genomics. EMBO reports 18, 1559-1571. https://doi.org/10.15252/embr.201744102

Hornung, P., Maier, M., Alushin, G.M., Lander, G.C., Nogales, E., Westermann, S., 2011. Molecular Architecture and Connectivity of the Budding Yeast Mtw1 Kinetochore Complex. Journal of Molecular Biology 405, 548-559. https://doi.org/10.1016/j.jmb.2010.11.012

Hornung, P., Troc, P., Malvezzi, F., Maier, M., Demianova, Z., Zimniak, T., Litos, G., Lampert, F., Schleiffer, A., Brunner, M., Mechtler, K., Herzog, F., Marlovits, T.C., Westermann, S., 2014. A cooperative mechanism drives budding yeast kinetochore assembly downstream of CENP-A. Journal of Cell Biology 206, 509524. https://doi.org/10.1083/jcb.201403081

Huis in 't Veld, P.J., Jeganathan, S., Petrovic, A., Singh, P., John, J., Krenn, V., Weissmann, F., Bange, T., Musacchio, A., 2016. Molecular basis of outer kinetochore assembly on CENP-T. eLife 5, e21007. https://doi.org/10.7554/eLife.21007

Janke, C., Magiera, M.M., Rathfelder, N., Taxis, C., Reber, S., Maekawa, H., Moreno-Borchart, A., Doenges, G., Schwob, E., Schiebel, E., Knop, M., 2004. A versatile toolbox for PCR-based tagging of yeast genes: new fluorescent proteins, more markers and promoter substitution cassettes. Yeast 21, 947-962. https://doi.org/10.1002/yea.1142

Jenni, S., Dimitrova, Y.N., Valverde, R., Hinshaw, S.M., Harrison, S.C., 2017. Molecular Structures of Yeast Kinetochore Subcomplexes and Their Roles in Chromosome Segregation. Cold Spring Harbor Symposia on Quantitative Biology 82, 83-89. https://doi.org/10.1101/sqb.2017.82.033738

Jenni, S., Harrison, S.C., 2018. Structure of the DASH/Dam1 complex shows its role at the yeast kinetochoremicrotubule interface. Science 360, 552-558. https://doi.org/10.1126/science.aar6436 
Jin, Q.W., Fuchs, J., Loidl, J., 2000. Centromere clustering is a major determinant of yeast interphase nuclear organization. J Cell Sci 113, 1903-1912.

Joglekar, A.P., Bloom, K., Salmon, E.D., 2009. In Vivo Protein Architecture of the Eukaryotic Kinetochore with Nanometer Scale Accuracy. Current Biology 19, 694-699. https://doi.org/10.1016/j.cub.2009.02.056

Joglekar, A.P., Bloom, K.S., Salmon, E., 2010. Mechanisms of force generation by end-on kinetochoremicrotubule attachments. Current Opinion in Cell Biology 22, 57-67. https://doi.org/10.1016/j.ceb.2009.12.010

Joglekar, A.P., Bouck, D.C., Molk, J.N., Bloom, K.S., Salmon, E.D., 2006. Molecular architecture of a kinetochore-microtubule attachment site. Nature cell biology 8, 581-585.

Joglekar, A.P., Salmon, E.D., Bloom, K.S., 2008. Counting Kinetochore Protein Numbers in Budding Yeast Using Genetically Encoded Fluorescent Proteins. Methods Cell Biol 85, 127-151. https://doi.org/10.1016/S0091-679X(08)85007-8

Keith, K.C., Fitzgerald-Hayes, M., 2000. CSE4 genetically interacts with the Saccharomyces cerevisiae centromere DNA elements CDE I and CDE II but not CDE III. Implications for the path of the centromere dna around a cse4p variant nucleosome. Genetics 156, 973-981.

Kim, S.J., Fernandez-Martinez, J., Nudelman, I., Shi, Y., Zhang, W., Raveh, B., Herricks, T., Slaughter, B.D., Hogan, J.A., Upla, P., Chemmama, I.E., Pellarin, R., Echeverria, I., Shivaraju, M., Chaudhury, A.S., Wang, J., Williams, R., Unruh, J.R., Greenberg, C.H., Jacobs, E.Y., Yu, Z., de la Cruz, M.J., Mironska, R., Stokes, D.L., Aitchison, J.D., Jarrold, M.F., Gerton, J.L., Ludtke, S.J., Akey, C.W., Chait, B.T., Sali, A., Rout, M.P., 2018. Integrative structure and functional anatomy of a nuclear pore complex. Nature 555, 475-482. https://doi.org/10.1038/nature26003

Klare, K., Weir, J.R., Basilico, F., Zimniak, T., Massimiliano, L., Ludwigs, N., Herzog, F., Musacchio, A., 2015. CENP-C is a blueprint for constitutive centromere-associated network assembly within human kinetochores. Journal of Cell Biology 210, 11-22. https://doi.org/10.1083/jcb.201412028

Krassovsky, K., Henikoff, J.G., Henikoff, S., 2012. Tripartite organization of centromeric chromatin in budding yeast. PNAS 109, 243-248. https://doi.org/10.1073/pnas.1118898109

Kudalkar, E.M., Scarborough, E.A., Umbreit, N.T., Zelter, A., Gestaut, D.R., Riffle, M., Johnson, R.S., MacCoss, M.J., Asbury, C.L., Davis, T.N., 2015. Regulation of outer kinetochore Ndc80 complex-based microtubule attachments by the central kinetochore Mis12/MIND complex. Proceedings of the National Academy of Sciences 112, E5583-E5589. https://doi.org/10.1073/pnas.1513882112

Lando, D., Endesfelder, U., Berger, H., Subramanian, L., Dunne, P.D., McColl, J., Klenerman, D., Carr, A.M., Sauer, M., Allshire, R.C., Heilemann, M., Laue, E.D., 2012. Quantitative single-molecule microscopy reveals that CENP-A ${ }^{\text {Cnp1 }}$ deposition occurs during G2 in fission yeast. Open Biology 2, 120078. https://doi.org/10.1098/rsob.120078

Lang, J., Barber, A., Biggins, S., 2018. An assay for de novo kinetochore assembly reveals a key role for the CENP-T pathway in budding yeast. eLife 7. https://doi.org/10.7554/eLife.37819

Lawrimore, J., Bloom, K.S., Salmon, E.D., 2011. Point centromeres contain more than a single centromerespecific Cse4 (CENP-A) nucleosome. The Journal of Cell Biology 195, 573-582.

Leber, V., Nans, A., Singleton, M.R., 2018. Structural basis for assembly of the CBF3 kinetochore complex. The EMBO Journal 37, 269-281. https://doi.org/10.15252/embj.201798134

Malvezzi, F., Litos, G., Schleiffer, A., Heuck, A., Mechtler, K., Clausen, T., Westermann, S., 2013. A structural basis for kinetochore recruitment of the Ndc80 complex via two distinct centromere receptors. The EMBO Journal 32, 409-423. https://doi.org/10.1038/emboj.2012.356

McEvoy, A.L., Hoi, H., Bates, M., Platonova, E., Cranfill, P.J., Baird, M.A., Davidson, M.W., Ewers, H., Liphardt, J., Campbell, R.E., 2012. mMaple: A Photoconvertible Fluorescent Protein for Use in Multiple Imaging Modalities. PloS one 7, e51314.

McIntosh, J.R., O’Toole, E., Zhudenkov, K., Morphew, M., Schwartz, C., Ataullakhanov, F.I., Grishchuk, E.L., 2013. Conserved and divergent features of kinetochores and spindle microtubule ends from five species. Journal of Cell Biology 200, 459-474. https://doi.org/10.1083/jcb.201209154

McKinley, K.L., Sekulic, N., Guo, L.Y., Tsinman, T., Black, B.E., Cheeseman, I.M., 2015. The CENP-L-N Complex Forms a Critical Node in an Integrated Meshwork of Interactions at the Centromere-Kinetochore Interface. Molecular Cell 60, 886-898. https://doi.org/10.1016/j.molcel.2015.10.027

Mizuguchi, G., Xiao, H., Wisniewski, J., Smith, M.M., Wu, C., 2007. Nonhistone Scm3 and Histones CenH3-H4 Assemble the Core of Centromere-Specific Nucleosomes. Cell 129, 1153-1164. https://doi.org/10.1016/j.cell.2007.04.026

Mund, M., van der Beek, J.A., Deschamps, J., Dmitrieff, S., Hoess, P., Monster, J.L., Picco, A., Nédélec, F., Kaksonen, M., Ries, J., 2018. Systematic Nanoscale Analysis of Endocytosis Links Efficient Vesicle Formation to Patterned Actin Nucleation. Cell 174, 884-896.e17. https://doi.org/10.1016/j.cell.2018.06.032 
Musacchio, A., Desai, A., 2017. A Molecular View of Kinetochore Assembly and Function. Biology 6, 5. https://doi.org/10.3390/biology6010005

Ng, R., Carbon, J., 1987. Mutational and in vitro protein-binding studies on centromere DNA from Saccharomyces cerevisiae. Molecular and Cellular Biology 7, 4522-4534. https://doi.org/10.1128/MCB.7.12.4522

Pekgöz Altunkaya, G., Malvezzi, F., Demianova, Z., Zimniak, T., Litos, G., Weissmann, F., Mechtler, K., Herzog, F., Westermann, S., 2016. CCAN Assembly Configures Composite Binding Interfaces to Promote CrossLinking of Ndc80 Complexes at the Kinetochore. Current Biology 26, 2370-2378. https://doi.org/10.1016/j.cub.2016.07.005

Pentakota, S., Zhou, K., Smith, C., Maffini, S., Petrovic, A., Morgan, G.P., Weir, J.R., Vetter, I.R., Musacchio, A., Luger, K., 2017. Decoding the centromeric nucleosome through CENP-N. elife 6. https://doi.org/10.7554/eLife.33442

Petrovic, A., Keller, J., Liu, Y., Overlack, K., John, J., Dimitrova, Y.N., Jenni, S., van Gerwen, S., Stege, P., Wohlgemuth, S., Rombaut, P., Herzog, F., Harrison, S.C., Vetter, I.R., Musacchio, A., 2016. Structure of the MIS12 Complex and Molecular Basis of Its Interaction with CENP-C at Human Kinetochores. Cell 167, 1028-1040.e15. https://doi.org/10.1016/j.cell.2016.10.005

Petrovic, A., Mosalaganti, S., Keller, J., Mattiuzzo, M., Overlack, K., Krenn, V., De Antoni, A., Wohlgemuth, S., Cecatiello, V., Pasqualato, S., Raunser, S., Musacchio, A., 2014. Modular Assembly of RWD Domains on the Mis12 Complex Underlies Outer Kinetochore Organization. Molecular Cell 53, 591-605. https://doi.org/10.1016/j.molcel.2014.01.019

Przewloka, M.R., Venkei, Z., Bolanos-Garcia, V.M., Debski, J., Dadlez, M., Glover, D.M., 2011. CENP-C Is a Structural Platform for Kinetochore Assembly. Current Biology 21, 399-405. https://doi.org/10.1016/j.cub.2011.02.005

Rieder, C.L., 1982. The Formation, Structure, and Composition of the Mammalian Kinetochore and Kinetochore Fiber, in: International Review of Cytology. Elsevier, pp. 1-58. https://doi.org/10.1016/S00747696(08)61672-1

Ries, J., 2020. SMAP: a modular super-resolution microscopy analysis platform for SMLM data. Nature Methods 17, 870-872. https://doi.org/10.1038/s41592-020-0938-1

Rust, M.J., Bates, M., Zhuang, X., 2006. Sub-diffraction-limit imaging by stochastic optical reconstruction microscopy (STORM). Nature Methods 3, 793-795. https://doi.org/10/fwrhq3

Santaguida, S., Amon, A., 2015. Short- and long-term effects of chromosome mis-segregation and aneuploidy. Nature Reviews Molecular Cell Biology 16, 473-485. https://doi.org/10.1038/nrm4025

Schmitzberger, F., Richter, M.M., Gordiyenko, Y., Robinson, C.V., Dadlez, M., Westermann, S., 2017. Molecular basis for inner kinetochore configuration through RWD domain-peptide interactions. The EMBO Journal 36, 3458-3482. https://doi.org/10.15252/embj.201796636

Scott, K.C., Bloom, K.S., 2014. Lessons learned from counting molecules: how to lure CENP-A into the kinetochore. Open Biology 4, 140191. https://doi.org/10.1098/rsob.140191

Screpanti, E., De Antoni, A., Alushin, G.M., Petrovic, A., Melis, T., Nogales, E., Musacchio, A., 2011. Direct Binding of Cenp-C to the Mis12 Complex Joins the Inner and Outer Kinetochore. Current Biology 21, 391-398. https://doi.org/10.1016/j.cub.2010.12.039

Shivaraju, M., Unruh, J.R., Slaughter, B.D., Mattingly, M., Berman, J., Gerton, J.L., 2012. Cell-Cycle-Coupled Structural Oscillation of Centromeric Nucleosomes in Yeast. Cell 150, 304-316. https://doi.org/10.1016/j.cell.2012.05.034

Sieben, C., Banterle, N., Douglass, K., Gonczy, P., Manley, S., 2018. Multicolor single particle reconstruction of protein complexes. bioRxiv 255364. https://doi.org/10.1101/255364

Sochacki, K.A., Dickey, A.M., Strub, M.-P., Taraska, J.W., 2017. Endocytic proteins are partitioned at the edge of the clathrin lattice in mammalian cells. Nature Cell Biology 19, 352-361. https://doi.org/10/f9vxd5

Sun, X., Zhang, A., Baker, B., Sun, L., Howard, A., Buswell, J., Maurel, D., Masharina, A., Johnsson, K., Noren, C.J., Xu, M.-Q., Corrêa, I.R., 2011. Development of SNAP-tag fluorogenic probes for wash-free fluorescence imaging. Chembiochem : a European journal of chemical biology 12, 2217-2226.

Szymborska, A., Marco, A. de, Daigle, N., Cordes, V.C., Briggs, J.A.G., Ellenberg, J., 2013. Nuclear Pore Scaffold Structure Analyzed by Super-Resolution Microscopy and Particle Averaging. Science 341, 655-658. https://doi.org/10.1126/science.1240672

Thevathasan, J.V., Kahnwald, M., Cieśliński, K., Hoess, P., Peneti, S.K., Reitberger, M., Heid, D., Kasuba, K.C., Hoerner, S.J., Li, Y., Wu, Y.-L., Mund, M., Matti, U., Pereira, P.M., Henriques, R., Nijmeijer, B., Kueblbeck, M., Sabinina, V.J., Ellenberg, J., Ries, J., 2019. Nuclear pores as versatile reference standards for quantitative superresolution microscopy. Nat Methods 16, 1045-1053. https://doi.org/10/gf85h3 
Ustinov, N.B., Korshunova, A.V., Gudimchuk, N.B., 2020. Protein Complex NDC80: Properties, Functions, and Possible Role in Pathophysiology of Cell Division. Biochemistry (Moscow) 85, 448-462. https://doi.org/10.1134/S0006297920040057

Valverde, R., Ingram, J., Harrison, S.C., 2016. Conserved Tetramer Junction in the Kinetochore Ndc80 Complex. Cell Reports 17, 1915-1922. https://doi.org/10.1016/j.celrep.2016.10.065

Virant, D., Vojnovic, I., Winkelmeier, J., Endesfelder, M., Turkowyd, B., Lando, D., Endesfelder, U., 2021. Unraveling the kinetochore nanostructure in Schizosaccharomyces pombe using multi-color singlemolecule localization microscopy. BioRxiv 469981.

Walstein, K., Petrovic, A., Pan, D., Hagemeier, B., Vogt, D., Vetter, I., Musacchio, A., 2020. Assembly principles and stoichiometry of a complete human kinetochore module. bioRxiv 2020.12.01.407130. https://doi.org/10.1101/2020.12.01.407130

Watanabe, R., Hara, M., Okumura, E., Hervé, S., Fachinetti, D., Ariyoshi, M., Fukagawa, T., 2019. CDK1mediated CENP-C phosphorylation modulates CENP-A binding and mitotic kinetochore localization. Journal of Cell Biology 218, 4042-4062. https://doi.org/10.1083/jcb.201907006

Wei, R.R., Al-Bassam, J., Harrison, S.C., 2007. The Ndc80/HEC1 complex is a contact point for kinetochoremicrotubule attachment. Nature Structural \& Molecular Biology 14, 54-59. https://doi.org/10.1038/nsmb1186

Wei, R.R., Sorger, P.K., Harrison, S.C., 2005. Molecular organization of the Ndc80 complex, an essential kinetochore component. Proceedings of the National Academy of Sciences 102, 5363-5367. https://doi.org/10.1073/pnas.0501168102

Winey, M., Mamay, C.L., O’Toole, E.T., Mastronarde, D.N., Giddings, T.H., Jr, McDonald, K.L., McIntosh, J.R., 1995. Three-dimensional ultrastructural analysis of the Saccharomyces cerevisiae mitotic spindle. Journal of Cell Biology 129, 1601-1615. https://doi.org/10.1083/jcb.129.6.1601

Wisniewski, J., Hajj, B., Chen, J., Mizuguchi, G., Xiao, H., Wei, D., Dahan, M., Wu, C., Kadonaga, J.T., 2014. Imaging the fate of histone Cse4 reveals de novo replacement in S phase and subsequent stable residence at centromeres. eLife 3 , e02203.

Xiao, H., Wang, F., Wisniewski, J., Shaytan, A.K., Ghirlando, R., FitzGerald, P.C., Huang, Y., Wei, D., Li, S., Landsman, D., Panchenko, A.R., Wu, C., 2017. Molecular basis of CENP-C association with the CENPA nucleosome at yeast centromeres. Genes \& Development 31, 1958-1972. https://doi.org/10.1101/gad.304782.117

Yan, K., Yang, J., Zhang, Z., McLaughlin, S.H., Chang, L., Fasci, D., Ehrenhofer-Murray, A.E., Heck, A.J.R., Barford, D., 2019. Structure of the inner kinetochore CCAN complex assembled onto a centromeric nucleosome. Nature 574, 278-282. https://doi.org/10.1038/s41586-019-1609-1

Yan, K., Zhang, Z., Yang, J., McLaughlin, S.H., Barford, D., 2018. Architecture of the CBF3-centromere complex of the budding yeast kinetochore. Nature Structural \& Molecular Biology 25, 1103-1110. https://doi.org/10.1038/s41594-018-0154-1

Zhang, W., Lukoyanova, N., Miah, S., Lucas, J., Vaughan, C.K., 2018. Insights into Centromere DNA Bending Revealed by the Cryo-EM Structure of the Core Centromere Binding Factor 3 with Ndc10. Cell Reports 24, 744-754. https://doi.org/10.1016/j.celrep.2018.06.068

Zinkowski, R.P., Meyne, J., Brinkley, B.R., 1991. The centromere-kinetochore complex: a repeat subunit model. Journal of Cell Biology 113, 1091-1110. https://doi.org/10.1083/jcb.113.5.1091 
bioRxiv preprint doi: https://doi.org/10.1101/2021.12.01.469648; this version posted December 3, 2021. The copyright holder for this

preprint (which was not certified by peer review) is the author/funder, who has granted bioRxiv a license to display the preprint in perpetuity. It is made available under aCC-BY-NC 4.0 International license.

\section{Supplementary Figures}

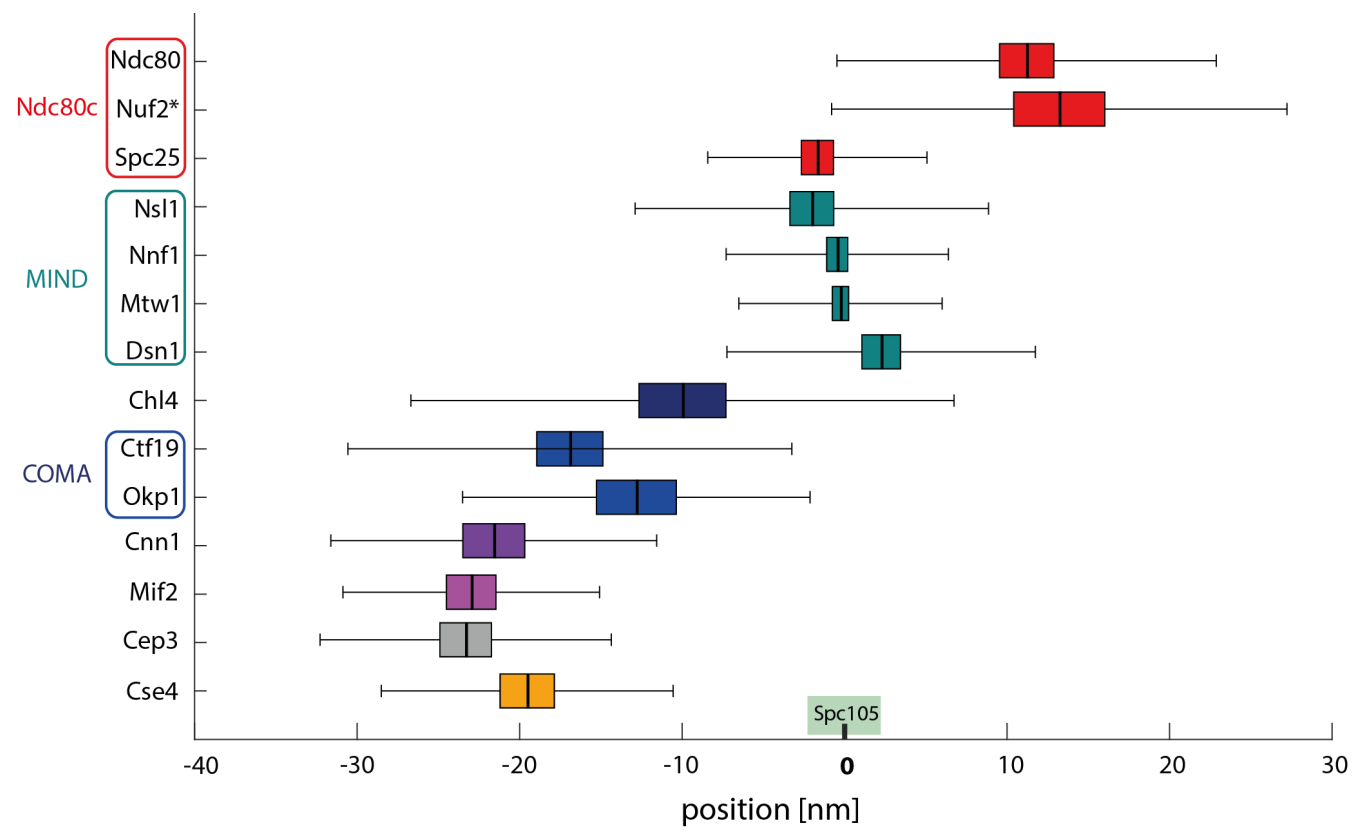

Figure S1. An independent analysis of intra-kinetochore distances based on manually picked single kinetochores. The mean distance is plotted with SEM (as colored box) and STD (whiskers).

A)
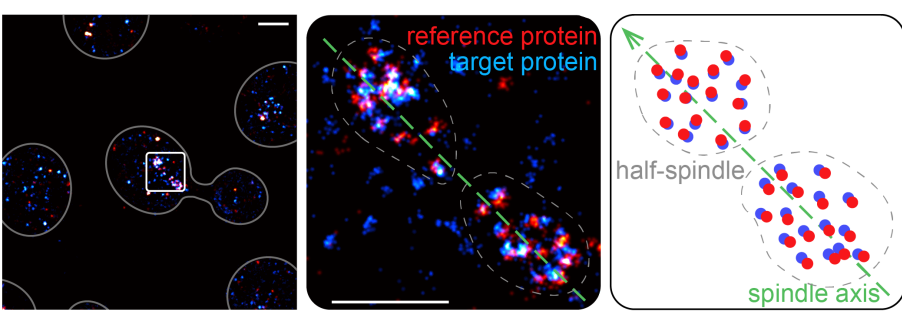

C)

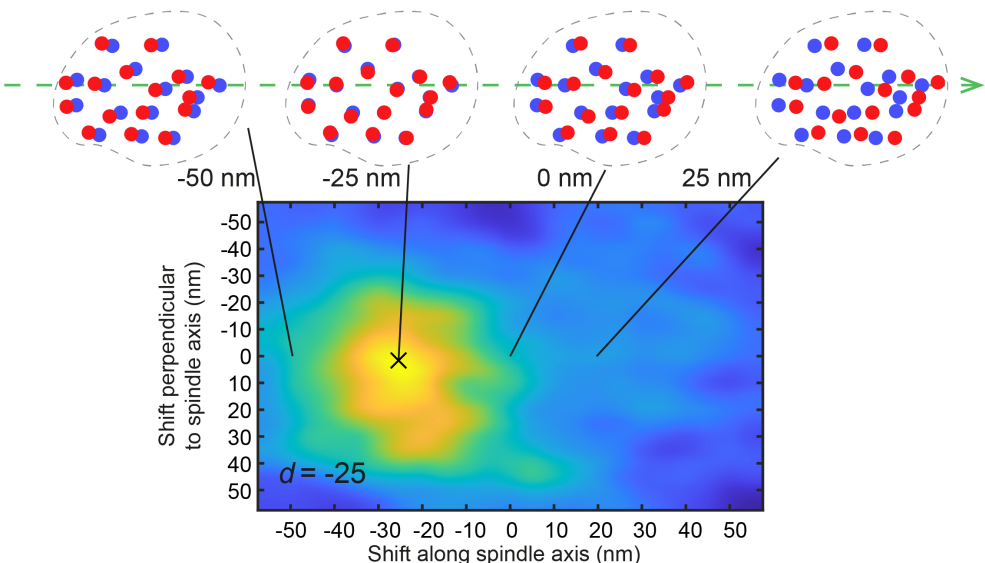

B)

Cross-correlation $\rightarrow$ protein distance

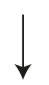

Combine pair of half-spindles

D)

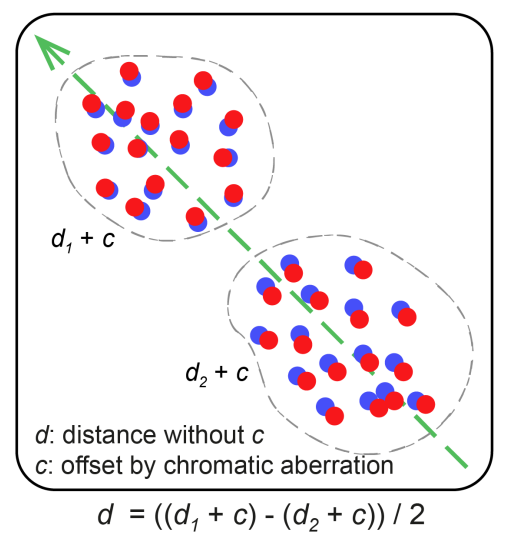

Figure S2. Workflow of quantifying the intrakinetochore distances. A) Metaphase spindles (white box) with both half spindles close to the focus are manually segmented (dashed contour). The spindle axis for each spindle is manually annotated (green dashed line). A schematic (right panel) is provided for clarity. B) The overview of the workflow. C) The intrakinetochore distance between the target and reference proteins is quantified using the cross-correlation analysis. This analysis is applied to each half-spindle and yields a correlation map showing the similarity between the two channels at certain lateral and axial shifts of the reference channel. The shift along spindle axis at the maximum is quantified as the intrakinetochore distance (d). D) To eliminate the potential offset $c$ caused by the chromatic aberration, the average intrakinetochore distances $d$ of both paired half-spindles, having the distances $d_{1}$ and $d_{2}$ respectively, is then calculated per spindle. 


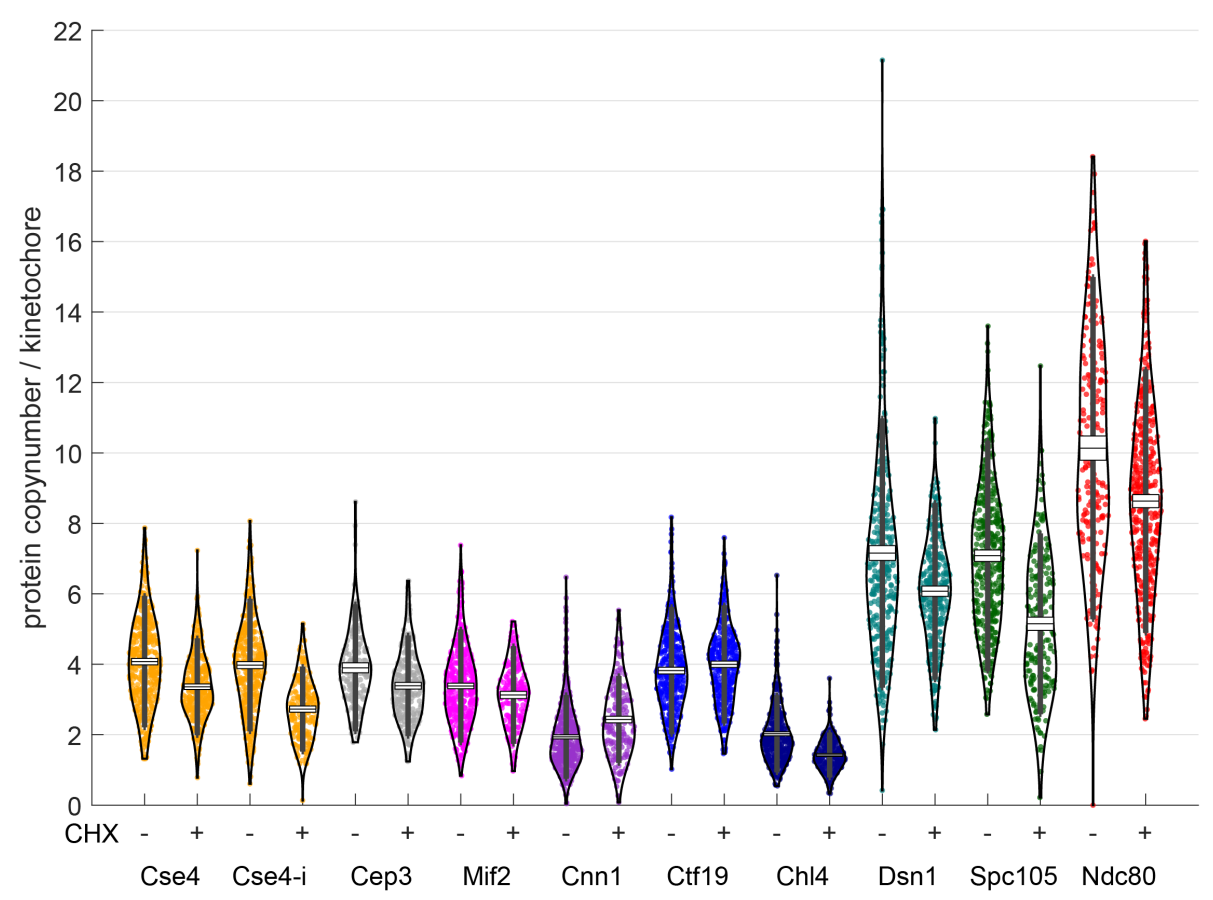

Figure S3. Protein copy numbers per kinetochore measured with and without cycloheximide (CHX) treatment (250 $\mathrm{ug} / \mathrm{ml}, 1 \mathrm{hr})$, respectively, to investigate the effect of protein maturation. Each data point corresponds to one kinetochore cluster. Boxes denote average copy numbers and standard error of means, and whiskers denote standard deviations.

\section{Supplementary Tables}

Table S1 The table represents the strains created and used in this study. All of them are based on the MKY100 strain (Kaksonen Lab) with the following genetic background: MATa, his34200, leu3-52, lys2-801.

\begin{tabular}{|c|c|}
\hline Strain name & Genotype \\
\hline Spc42-GFP/Spc105-SNAP/Cep3-mMaple & $\begin{array}{l}\text { SPC42-GFP::kanMX4, SPC105-SNAP::hphNT1,CEP3- } \\
\text { mMaple::HIS3MX6 }\end{array}$ \\
\hline Spc42-GFP/Spc105-SNAP/Cse4-mMaple & $\begin{array}{l}\text { SPC42-GFP::kanMX4, SPC105-SNAP::hphNT1,CSE4- } \\
\text { mMaple::HIS3MX6 }\end{array}$ \\
\hline Spc42-GFP/Spc105-SNAP/Cnn1-mMaple & $\begin{array}{l}\text { SPC42-GFP::kanMX4, SPC105-SNAP::hphNT1,CNN1- } \\
\text { mMaple::HIS3MX6 }\end{array}$ \\
\hline Spc42-GFP/Spc105-SNAP/Chl4-mMaple & $\begin{array}{l}\text { SPC42-GFP::kanMX4, SPC105-SNAP::hphNT1,CHL4- } \\
\text { mMaple::HIS3MX6 }\end{array}$ \\
\hline Spc42-GFP/Spc105-SNAP/Ctf19-mMaple & $\begin{array}{l}\text { SPC42-GFP::kanMX4, SPC105-SNAP::hphNT1,CTF19- } \\
\text { mMaple::HIS3MX6 }\end{array}$ \\
\hline Spc42-GFP/Spc105-SNAP/Dsn1-mMaple & $\begin{array}{l}\text { SPC42-GFP::kanMX4, SPC105-SNAP::hphNT1,DSN1- } \\
\text { mMaple::HIS3MX6 }\end{array}$ \\
\hline Spc42-GFP/Spc105-SNAP/Mif2-mMaple & $\begin{array}{l}\text { SPC42-GFP::kanMX4, SPC105-SNAP::hphNT1,MIF2- } \\
\text { mMaple::HIS3MX6 }\end{array}$ \\
\hline Spc42-GFP/Spc105-SNAP/Mtw1-mMaple & $\begin{array}{l}\text { SPC42-GFP::kanMX4, SPC105-SNAP::hphNT1,MTW1- } \\
\text { mMaple::HIS3MX6 }\end{array}$ \\
\hline Spc42-GFP/Spc105-SNAP/Ndc80-mMaple & $\begin{array}{l}\text { SPC42-GFP::kanMX4, SPC105-SNAP::hphNT1,NDC80- } \\
\text { mMaple::HIS3MX6 }\end{array}$ \\
\hline Spc42-GFP/Spc105-SNAP/Nnf1-mMaple & $\begin{array}{l}\text { SPC42-GFP::kanMX4, SPC105-SNAP::hphNT1,NNF1- } \\
\text { mMaple::HIS3MX6 }\end{array}$ \\
\hline Spc42-GFP/Spc105-SNAP/Ns11-mMaple & $\begin{array}{l}\text { SPC42-GFP::kanMX4, SPC105-SNAP::hphNT1,NSL1- } \\
\text { mMaple::HIS3MX6 }\end{array}$ \\
\hline Spc42-GFP/Spc105-SNAP/Spc25-mMaple & $\begin{array}{l}\text { SPC42-GFP::kanMX4, SPC105-SNAP::hphNT1,SPC25- } \\
\text { mMaple::HIS3MX6 }\end{array}$ \\
\hline Spc42-GFP/Ndc80-SNAP/Ctf19-mMaple & $\begin{array}{l}\text { SPC42-GFP::kanMX4, SPC105-SNAP::hphNT1,CTF19- } \\
\text { mMaple::HIS3MX6 }\end{array}$ \\
\hline
\end{tabular}




\begin{tabular}{l|l} 
Ndc80-SNAP/Nuf2-mMaple & NDC80-SNAP::hphNT1, NUF2-mMaple::HIS3MX6 \\
\hline Ndc80-GFP/Cep3-mMaple & NDC80-GFP::kanMX4, CEP3-mMaple::HIS3MX6 \\
\hline Ndc80-GFP/Cse4-mMaple & NDC80-GFP::kanMX4, CSE4-mMaple::HIS3MX6 \\
\hline Ndc80-GFP/Cse4-mMaple-Cse4 & NDC80-GFP::kanMX4, cse4::CSE4-mMaple-CSE4::HIS3MX6 \\
\hline Ndc80-GFP/Cnn1-mMaple & NDC80-GFP::kanMX4, CNN1-mMaple::HIS3MX6 \\
\hline Ndc80-GFP/Ch14-mMaple & NDC80-GFP::kanMX4, CHL4-mMaple::HIS3MX6 \\
\hline Ndc80-GFP/Ctf19-mMaple & NDC80-GFP::kanMX4, CTF19-mMaple::HIS3MX6 \\
\hline Ndc80-GFP/Dsn1-mMaple & NDC80-GFP::kanMX4, DSN1-mMaple::HIS3MX6 \\
\hline Ndc80-GFP/Mif2-mMaple & NDC80-GFP::kanMX4, MIF2-mMaple::HIS3MX6 \\
\hline Ndc80-GFP/Mtw1-mMaple & NDC80-GFP::kanMX4, MTW1-mMaple::HIS3MX6 \\
\hline Spc42-GFP/Ndc80-mMaple & SPC42-GFP::kanMX4, NDC80-mMaple::HIS3MX6 \\
\hline Ndc80-GFP/ Nnf1-mMaple & NDC80-GFP::kanMX4, NNF1-mMaple::HIS3MX6 \\
\hline Ndc80-GFP/ Ns11-mMaple & NDC80-GFP::kanMX4, NSL1-mMaple::HIS3MX6 \\
\hline Ndc80-GFP/ Spc25-mMaple & NDC80-GFP::kanMX4, SPC25-mMaple::HIS3MX6 \\
\hline Abp1-GFP/Nup188-mMaple & ABP1-GFP::kanMX4, NUP188-mMaple:HIS3MX6
\end{tabular}

Table S2 Shown in this table are further information about the dual-color SMLM experiments. For each protein of interest, the number of performed experiments, ROIs and kinetochore spindles are depicted.

\begin{tabular}{c|c|c|c} 
Protein & $\begin{array}{l}\text { Number of } \\
\text { experiments }\end{array}$ & $\begin{array}{l}\text { Number of } \\
\text { ROIs }\end{array}$ & $\begin{array}{l}\text { Number of } \\
\text { kinetochore spindles }\end{array}$ \\
\hline Ndc80 & 21 & 48 & 24 \\
\hline Nuf2* & 12 & 14 & 7 \\
\hline Spc25 & 26 & 26 & 11 \\
\hline Ns11 & 8 & 22 & 11 \\
\hline Nnf1 & 10 & 22 & 17 \\
\hline Mtw1 & 23 & 34 & 14 \\
\hline Dsn1 & 15 & 28 & 16 \\
\hline Ch14 & 22 & 32 & 17 \\
\hline Ctf19 & 12 & 34 & 10 \\
\hline Okp1 & 29 & 20 & 10 \\
\hline Cnn1 & 11 & 14 & 10 \\
\hline Mif2 & 13 & 20 & 9 \\
\hline Cep3 & 9 & 20 & 29
\end{tabular}

